\title{
FROM COMBINATORIAL GAMES TO SHAPE-SYMMETRIC MORPHISMS
}

\section{Michel Rigo}

http://www.discmath.ulg.ac.be/

http://orbi.ulg.ac.be/

Jean-Morlet chair: Shigeki Akiyama

Research school: Tiling Dynamical System, 20-24 November 2017

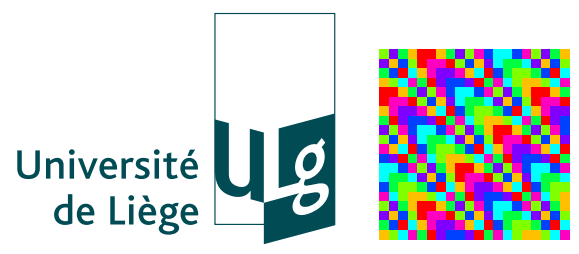




\section{Tentative OUtLINE OF THE TALKS}

Lecture 1:

- Starting from CGT

- Recap on $k$-automatic sequences

Lecture 2:

- Moving to multidimensional sequences

- Motivations from CGT: Wythoff's $\mathcal{P}$-positions

- Maes' shape-symmetric morphisms

Lecture 3:

- Link with (abstract) numeration systems

- Back to CGT and Presburger definable sets, work in progress 


\section{Notation AND CONVENTIONS}

- MSDF most significant digit first (on the left)

- $k \geq 2$ is an integer

- $\operatorname{rep}_{2}(81)=1010001 ; \operatorname{rep}_{k}$ canonical base- $k$ representation

- numerical value

$$
\operatorname{val}_{k}\left(d_{\ell} \cdots d_{0}\right)=\sum_{j=0}^{\ell} d_{j} k^{j}
$$

- extension to another system $S: \operatorname{rep}_{S}$ and $\operatorname{val}_{S}$

- $\mathbb{N}=\{0,1,2, \ldots\}$

- an infinite word $w: \mathbb{N} \rightarrow A$ (indexing starts with 0 ) 


\section{LET'S TRY TO MIX TWO TOPICS}

$\rightsquigarrow$ Motivations from Combinatorial Game Theory (CGT)

- independent community of researchers

- use tools from number theory, numeration systems, continued fractions, combinatorics on words, cellular automata, etc.

Some general references in CGT:

- A. Siegel, Combinatorial game theory, Graduate Studies in Mathematics, 146, AMS (2013). [1]

- E. R. Berlekamp, J. H. Conway, R. K. Guy, Winning ways for your mathematical plays, A K Peters, Ltd., (2001). [2]

- T. S. Ferguson, Game Theory, UCLA. [3] 
Wythoff's game [5] or, the Queen 紫 goes to $(0,0)$

- two players playing alternatively;

- the player unable to move loses the game (Normal play);

- two piles of token;

- Nim rule [6]: remove a positive number of token from one pile 莺

$$
\text { Moves }=\{(i, 0),(0, i) \mid i \geq 1\} .
$$

- Wythoff's rule: remove simultaneously the same number of token from both piles

$$
\text { Moves }=\{(i, 0),(0, i),(i, i) \mid i \geq 1\} .
$$


紫 $(6,3)$

0,0

6,3

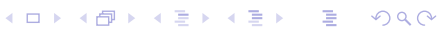


柴 $(6,3)$

$0,3,3,3,4,3,5,3) 6,3$ 


$$
\text { 紫 }(6,3) \stackrel{A}{\longrightarrow}(4,1)
$$

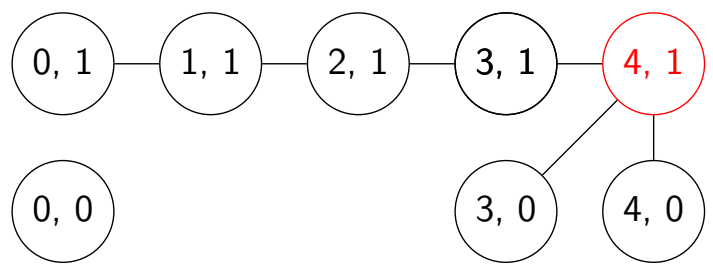




$$
\text { 觜 }(6,3) \stackrel{A}{\longrightarrow}(4,1) \stackrel{B}{\longrightarrow}(2,1)
$$

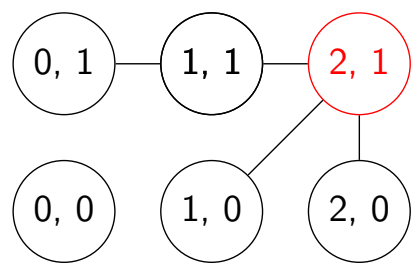




$$
\text { 紫 }(6,3) \stackrel{A}{\longrightarrow}(4,1) \stackrel{B}{\longrightarrow}(2,1) \stackrel{A}{\longrightarrow}(1,0)
$$

$$
\text { 6, } 3
$$
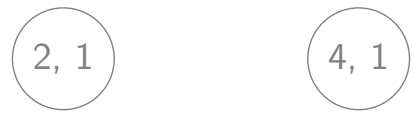

$0,0-1,0$ 


$$
\text { 觜 }(6,3) \stackrel{A}{\longrightarrow}(4,1) \stackrel{B}{\longrightarrow}(2,1) \stackrel{A}{\longrightarrow}(1,0) \stackrel{B}{\longrightarrow}(0,0)
$$

$$
6,3
$$
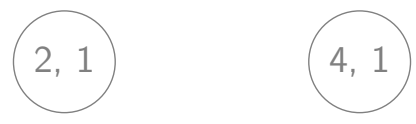

$0,01,0$ 


\section{WINNING AND LOSING POSITIONS}

\section{StATUS $\mathcal{N}$ (NEXT) OR P (PREVIOUS)}

A position is $\mathcal{P}$, if all its options are $\mathcal{N}$;

A position is $\mathcal{N}$, if there exists an option in $\mathcal{P}$.

If the game-graph:

- vertices $=$ positions

- edges = available options

is acyclic, then every position is either $\mathcal{N}$, or $\mathcal{P}$.

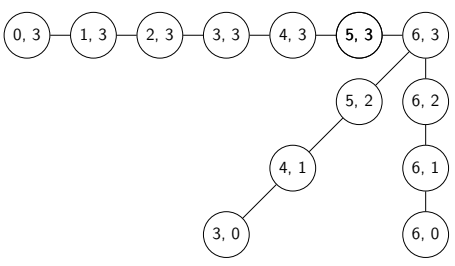

\section{REMARK (GRAPH-THEORETIC NOTION)}

- The set of $\mathcal{P}$-positions is the kernel of the game-graph [4].

- The game-graph grows exponentially.

A winning strategy is a map from $\mathcal{N}$ to $\mathcal{P}$ assigning to every winning position in $\mathcal{N}$ an available option in $\mathcal{P}$. 


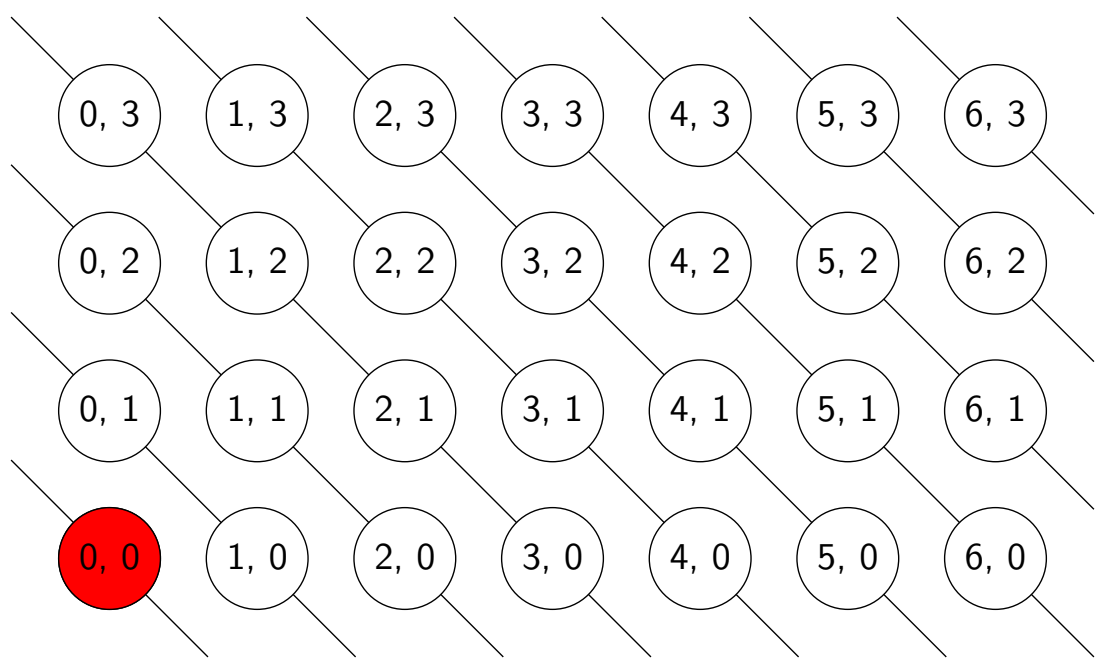

$\mathcal{P}$-positions and $\mathcal{N}$-positions for Wythoff's game. 


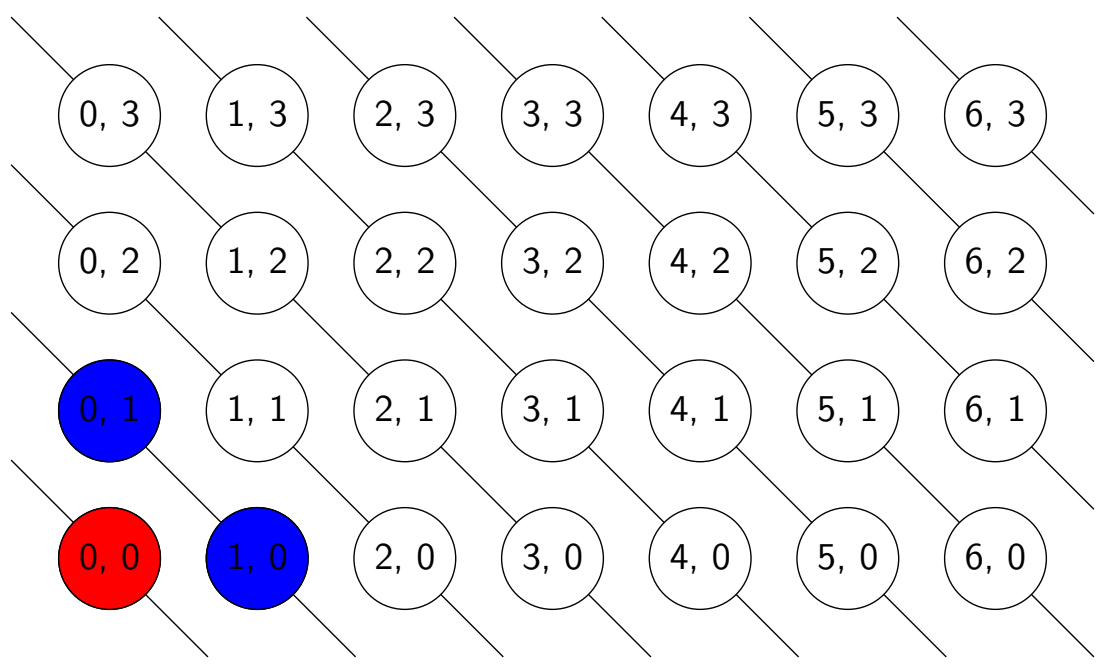

$\mathcal{P}$-positions and $\mathcal{N}$-positions for Wythoff's game. 


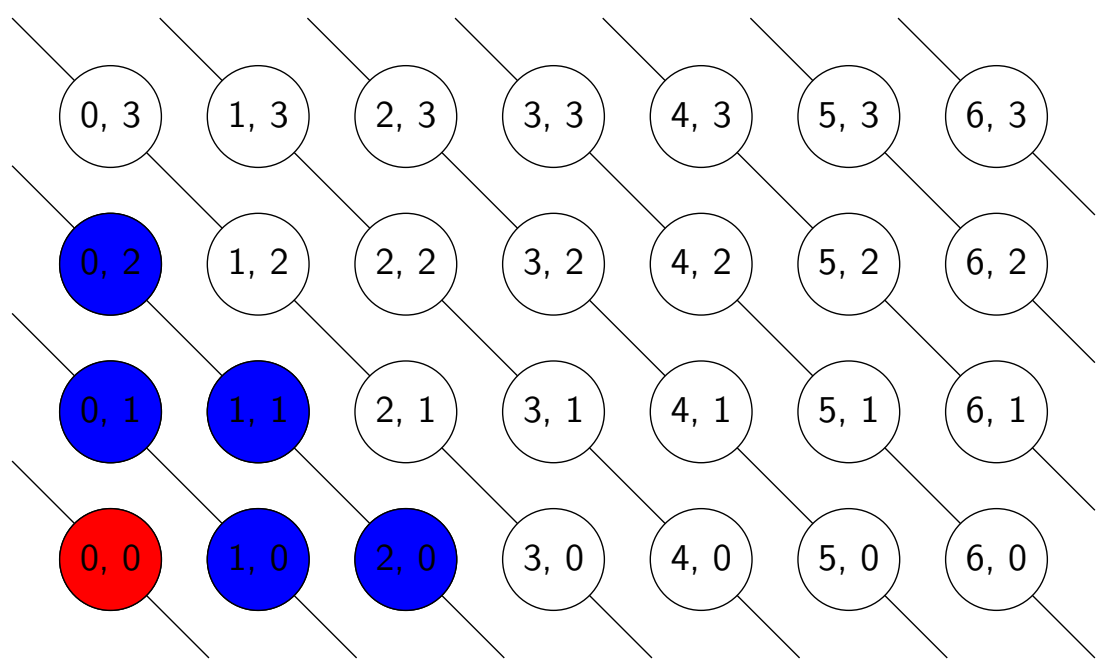

$\mathcal{P}$-positions and $\mathcal{N}$-positions for Wythoff's game. 


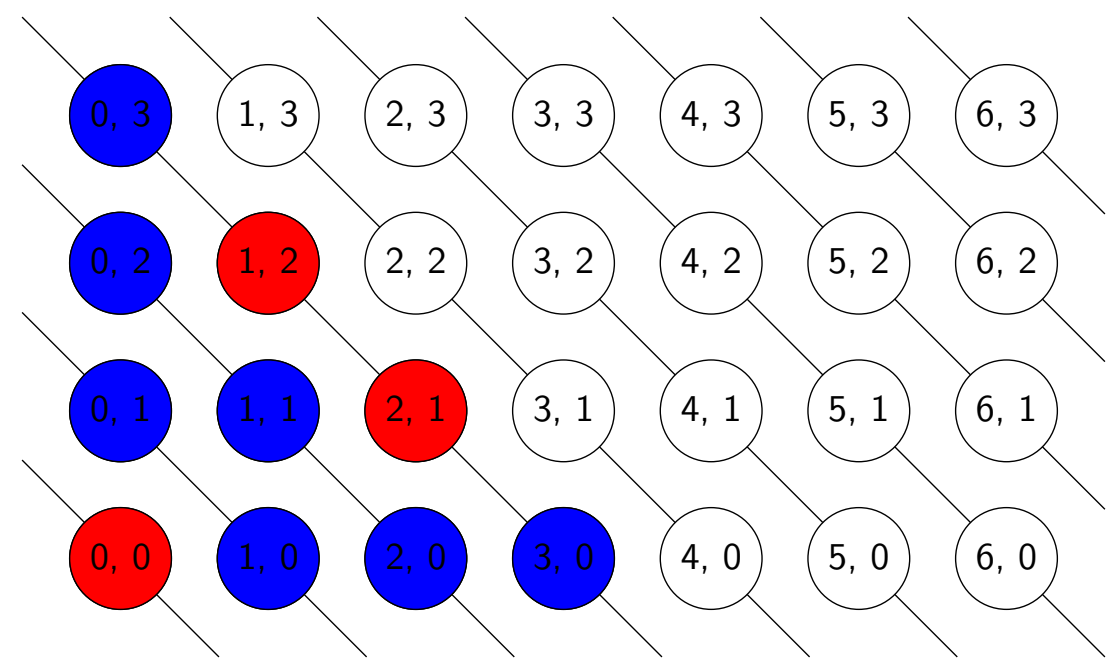

$\mathcal{P}$-positions and $\mathcal{N}$-positions for Wythoff's game. 


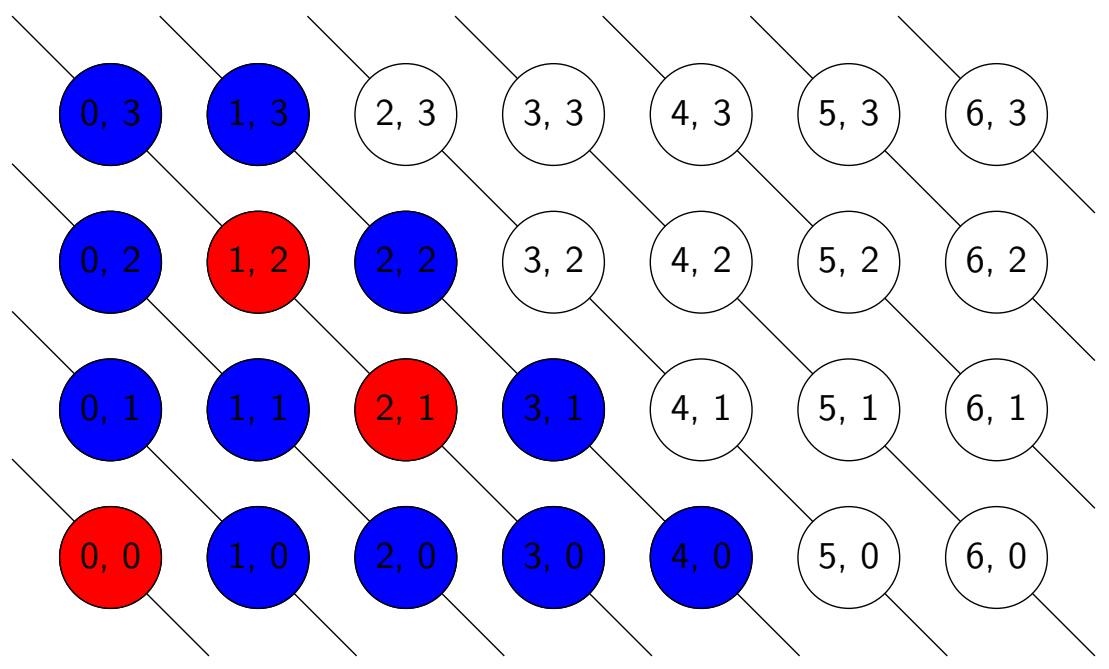

$\mathcal{P}$-positions and $\mathcal{N}$-positions for Wythoff's game. 


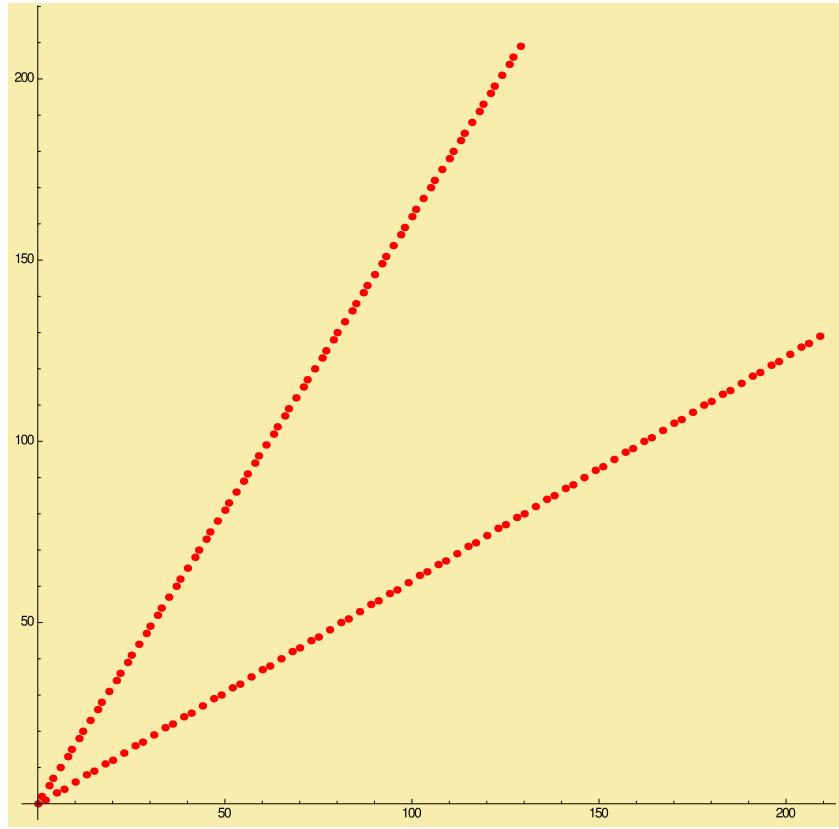

$\mathcal{P}$-positions and $\mathcal{N}$-positions for Wythoff's game. 
General questions

- Characterize the set of $\mathcal{P}$-positions?

- Is it computationally hard to determine these positions?

- Compute a winning strategy.

THeOREM (W. A. WYTHOPF, 1907 


\section{General questions}

- Characterize the set of $\mathcal{P}$-positions?

- Is it computationally hard to determine these positions?

- Compute a winning strategy.

\section{Theorem (W. A. Wythoff, 1907 [5])}

$(x, y)$ is a $\mathcal{P}$-position if and only if it is of the form

$$
\left(\lfloor n \varphi\rfloor,\left\lfloor n \varphi^{2}\right\rfloor\right), \quad \text { for some } n
$$

where $\varphi$ is the Golden mean.

Several other characterizations are known. $\rightsquigarrow$ Exercise: try to prove the above result (we will do it for $\mathrm{Nim}$ ). 


\section{WinNing STRATEGY FOR NIM : XOR OR NIM-SUM}

$7 \oplus 2 \oplus 9=12$

\begin{tabular}{llll} 
& 1 & 1 & 1 \\
& & 1 & 0 \\
1 & 0 & 0 & 1 \\
\hline 1 & 1 & 0 & 0
\end{tabular}

\section{Theorem (L. Bouton, 1902 [6])}

For the game of Nim with $n$ piles of token, a position $\left(x_{1}, \ldots, x_{n}\right)$ is in $\mathcal{P}$ if and only if

$$
\bigoplus_{i=1}^{n} x_{i}=0 \text {. }
$$

Exercise: $(\mathbb{N}, \oplus)$ is an abelian group. 
Proof.

1) $(0, \ldots, 0)$ is in $\mathcal{P}$.

2) If a position has a zero Nim-sum, all its options have a non-zero Nim-sum

$$
\begin{array}{llll}
1 & 0 & 1 & 1 \\
& & 1 & 0 \\
1 & 0 & 0 & 1 \\
\hline 0 & 0 & 0 & 0
\end{array}
$$

On a single row, one changes at least one digit $\rightsquigarrow$ non-zero sum. 
3) If a position has a non-zero Nim-sum, there is an option having a zero Nim-sum

$$
\begin{array}{llll} 
& 1 & 1 & 1 \\
& & 1 & 0 \\
1 & 0 & 0 & 1 \\
\hline 1 & 1 & 0 & 0
\end{array}
$$


3) If a position has a non-zero Nim-sum, there is an option having a zero Nim-sum

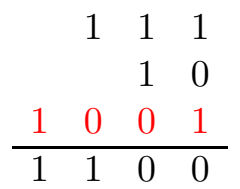


3) If a position has a non-zero Nim-sum, there is an option having a zero Nim-sum

$$
\begin{array}{llll} 
& 1 & 1 & 1 \\
& & 1 & 0 \\
0 & 1 & 0 & 1 \\
\hline 0 & 0 & 0 & 0
\end{array}
$$




\section{GRUNDY FUNCTION (FOR SUMS OF GAMES)}

\section{DEFINITION}

Let $S \subset \mathbb{N}$. MeX (minimum excluded value) of $S=\min \mathbb{N} \backslash S$.

Let $G$ be a combinatorial game and $x$ be a position.

The Grundy function is given by

$$
\mathcal{G}(x)=\operatorname{MeX}(\mathcal{G}(\operatorname{Opt}(x))) .
$$

$$
\operatorname{MeX}\{0,1,3,5\}=2, \quad \operatorname{MeX}\{2,3,6\}=0 .
$$

We have a characterization of the $\mathcal{P}$-positions:

\section{IMPORTANT OBSERVATION}

MeX $\emptyset=0$, thus $\mathcal{G}(x)=0$ iff $x$ is in $\mathcal{P}$. 


\section{GRUNDY FUNCTION (FOR SUMS OF GAMES)}

\section{Theorem (Sprague-Grundy [3])}

Let $G_{i}$ be combinatorial games with $\mathcal{G}_{i}$ as Grundy function, $i=1, \ldots, n$. Then $G_{1}+\cdots+G_{n}$ has Grundy function

$$
\mathcal{G}\left(x_{1}, \ldots, x_{n}\right)=\mathcal{G}_{1}\left(x_{1}\right) \oplus \cdots \oplus \mathcal{G}_{n}\left(x_{n}\right) .
$$

\section{APPLICATION}

Let's play on four boards simultaneously:

- $G_{1} \operatorname{Nim} \mathcal{G}_{1}(2,5)=7$

- $G_{2}$ Wythoff $\mathcal{G}_{2}(3,4)=2$

- $G_{3}$ Nim on three piles $\mathcal{G}_{3}(8,7,6)=9$

- $G_{4}$ Wythoff $\mathcal{G}_{4}(3,9)=12$

Should you start? Just compute whether $7 \oplus 2 \oplus 9 \oplus 12$ is 0 or not? 
General questions (one more)

- Characterize the set of $\mathcal{P}$-positions?

- Is it computationally hard to determine these positions?

- Compute a winning strategy.

Thanks to Sprague-Grundy theorem, we have an extra motivation:

- Compute the Grundy function of all positions. 
For the game of Nim, first few values of $(x, y) \mapsto \mathcal{G}_{N}(x, y)=x \oplus y$

\begin{tabular}{c|ccccccccccc}
$\vdots$ & $\vdots$ & & & & & & & & & & $\vdots$ \\
9 & 9 & 8 & 11 & 10 & 13 & 12 & 15 & 14 & 1 & 0 & \\
8 & 8 & 9 & 10 & 11 & 12 & 13 & 14 & 15 & 0 & 1 & \\
7 & 7 & 6 & 5 & 4 & 3 & 2 & 1 & 0 & 15 & 14 & \\
6 & 6 & 7 & 4 & 5 & 2 & 3 & 0 & 1 & 14 & 15 & \\
5 & 5 & 4 & 7 & 6 & 1 & 0 & 3 & 2 & 13 & 12 & \\
4 & 4 & 5 & 6 & 7 & 0 & 1 & 2 & 3 & 12 & 13 & \\
3 & 3 & 2 & 1 & 0 & 7 & 6 & 5 & 4 & 11 & 10 & \\
2 & 2 & 3 & 0 & 1 & 6 & 7 & 4 & 5 & 10 & 11 & \\
1 & 1 & 0 & 3 & 2 & 5 & 4 & 7 & 6 & 9 & 8 & \\
0 & 0 & 1 & 2 & 3 & 4 & 5 & 6 & 7 & 8 & 9 & $\cdots$ \\
\hline & 0 & 1 & 2 & 3 & 4 & 5 & 6 & 7 & 8 & 9 & $\cdots$
\end{tabular}

$\rightsquigarrow$ Exercises 21 and 22 in Section 16.6, p.451, Allouche-Shallit'03 [7].

What can be said about the structure of this table? 


\section{$k$-AUTOMATIC AND $k$-REGULAR SEQUENCES}

There are various characterizations of $k$-automatic sequences:

- Image under a coding of a fixed point of a $k$-uniform morphism;

- Sequence of outputs of a DFAO fed with base- $k$ expansions;

- Sets of indices defined by a first order formula in $\left\langle\mathbb{N},+, V_{k}\right\rangle$;

- $p$-algebraic formal power series;

- Column of the space-time diagram of a cellular automaton;

- Finiteness of the $k$-kernel.

A. Cobham 1972 [8], Büchi-Bruyère theorem 1960-1985 [9],

Christol-Kamae-Mendès France-Rauzy 1980 [12, 13], Rowland-Yassawi 2015 [14], S. Eilenberg 1974 [15, 11].

$\rightsquigarrow$ see the survey by Bruyère, Hansel, Michaux and Villemaire [10]. 


\section{Illustration $1 / 3$}

- Image under a coding of a fixed point of a $k$-uniform morphism;

- Sequence of outputs of a DFAO fed with base- $k$ expansions;

$$
f:\left\{\begin{aligned}
\mathrm{a} & \mapsto \mathrm{abc} \\
\mathrm{b} & \mapsto \mathrm{cbc} \\
\mathrm{c} & \mapsto \mathrm{bca}
\end{aligned}\right.
$$

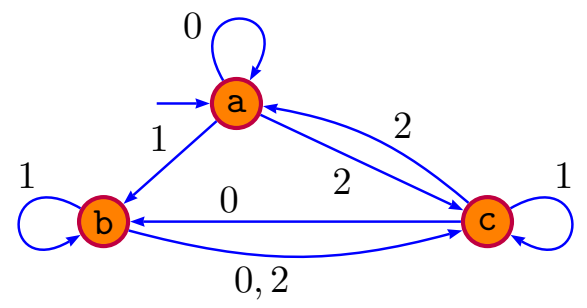

$f^{\omega}(\mathrm{a})=$ abccbcbcabcacbcbcacbcbcaabc $\ldots$

$g\left(f^{\omega}(\mathrm{a})\right)=100000001001000001000001100 \cdots$ 
Let $f: A^{*} \rightarrow A^{*}$ such that $|f(a)|=k$ for all $a \in A$.

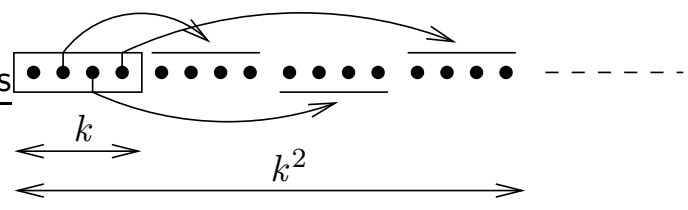

Figure: Constant length morphism $k=4$.

$$
\mathbf{x}=f^{\omega}(a)=x_{0} x_{1} x_{2} \quad \cdots x_{q} \cdots \quad \cdots x_{j} \cdots \quad \cdots
$$

\section{FUNDAMENTAL LEMMA (EXERCISE) [8]}

Let $j, k^{m} \leq j<k^{m+1}$. We have $j=k q+r, k^{m-1} \leq q<k^{m}$ and $0 \leq r<k$. The symbol $x_{j}$ is the $(r+1)$ th symbol in $f\left(x_{q}\right)$. 
Keeping track of the past - Desubstitution $k=3$

$$
\begin{aligned}
& a \\
& \text { a b c } \\
& \begin{array}{llllllllll}
a & b & c & c & b & c & b & c & a
\end{array} \\
& \begin{array}{llllllllllllllllllll}
a & b & c & c & b & c & b & c & a & b & c & a & c & b & c & b & c & a & \cdots
\end{array} \\
& \begin{array}{lllll}
0 & 1 & 2 & 3 & 4
\end{array} \\
& 14=3.4+2 \text { i.e. } c \text { is the third letter in } f\left(x_{4}\right)=f(b)=c b c \\
& 4=3.1+1 \text { i.e. } x_{4} \text { is the second letter in } f\left(x_{1}\right)=f(b)=c b c \\
& \operatorname{rep}_{2}(14)=112 \text {. }
\end{aligned}
$$


Keeping track of the past - Desubstitution $k=3$

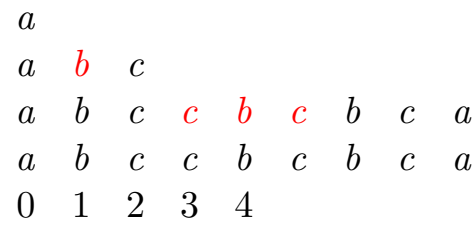

$14=3.4+2$ i.e. $c$ is the third letter in $f\left(x_{4}\right)=f(b)=c b c$

$4=3.1+1$ i.e. $x_{4}$ is the second letter in $f\left(x_{1}\right)=f(b)=c b c$

$$
\operatorname{rep}_{2}(14)=112 \text {. }
$$

Exercise: prove that the factor complexity of a $k$-automatic word $\mathbf{x}$ is in $\mathcal{O}(n)$. Hint: For $n \in\left(k^{\ell-1}, k^{\ell}\right]$, each factor of length $n$ is inside $x\left(j k^{\ell}\right) \cdots x\left((j+2) k^{\ell}-1\right)$ for some $j$. [8] 


\section{Illustration $2 / 3$}

- Sequence of outputs of a DFAO fed with base- $k$ expansions;

- Sets of indices defined by a first order formula in $\left\langle\mathbb{N},+, V_{k}\right\rangle$;

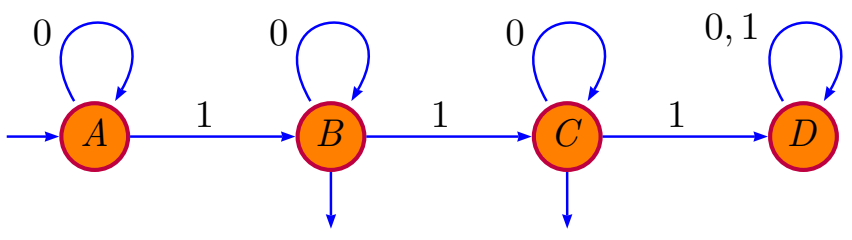

$$
\begin{gathered}
X=\{1,2,3,4,5,6,8,9,10,12,16,17,18,20,24, \ldots\} \\
\operatorname{rep}_{2}(X)=\{1,10,11,100,101,110,1000,1001,1010,1100, \ldots\} \\
X=\left\{n \in \mathbb{N} \mid \exists i, j \geq 0: n=2^{i}+2^{j}\right\} \cup\{1\}
\end{gathered}
$$$$
\psi(n) \equiv(n=1) \vee(\exists i)(\exists j)\left(V_{2}(i)=i \wedge V_{2}(j)=j \wedge n=i+j\right) .
$$ 


\section{Illustration 3/3}

- Rowland-Yassawi: A sequence over a finite field $\mathbb{F}_{q}$ of characteristic $p$ is $p$-automatic if and only if it occurs as a column of the space-time diagram, with eventually periodic initial conditions, of a linear cellular automaton with memory over $\mathbb{F}_{q} \cdot[14]$

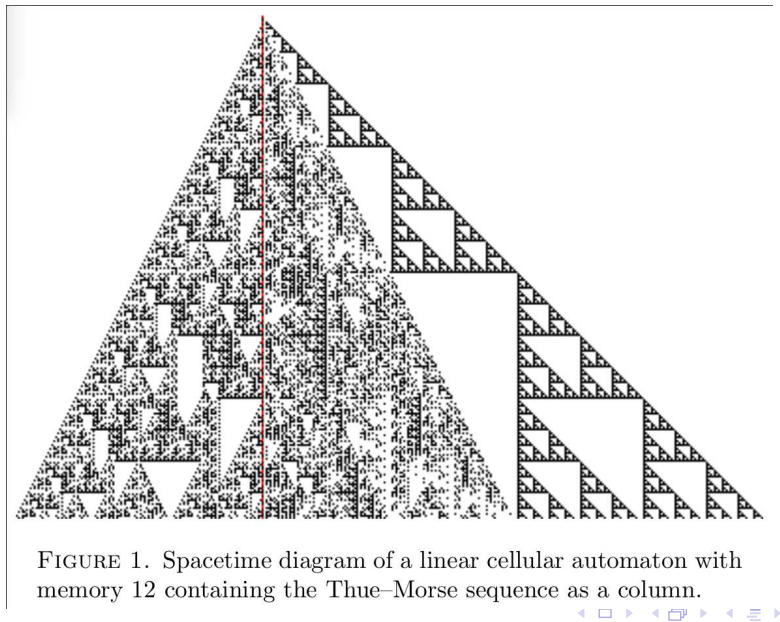




\section{DEFINITION [15]}

The $k$-kernel of a sequence $\mathbf{x}=(x(n))_{n \geq 0}$ is a set of subsequences:

$$
\operatorname{Ker}_{k}(\mathbf{x})=\left\{\left(x\left(k^{i} n+s\right)\right)_{n \geq 0} \mid i \geq 0,0 \leq s<k^{i}\right\} .
$$

Alternative definition, introduce $k$ operators of $k$-decimation, $r \in\{0, \ldots, k-1\}$,

$$
\partial_{k, r}\left((x(n))_{n \geq 0}\right)=(x(k n+r))_{n \geq 0} .
$$

Thus the $k$-kernel is the set of sequences of the form

$$
\partial_{k, r_{1}} \circ \cdots \circ \partial_{k, r_{m}}\left((x(n))_{n \geq 0}\right) .
$$

\section{REMARK}

This corresponds to selecting a suffix in base- $k$ expansions.

$$
\operatorname{val}_{k}\left(0^{i-m} r_{m} \cdots r_{1}\right)=s .
$$


Some of these subsequences; suffixes: $\varepsilon, 0,1,00,01,10,11,000$
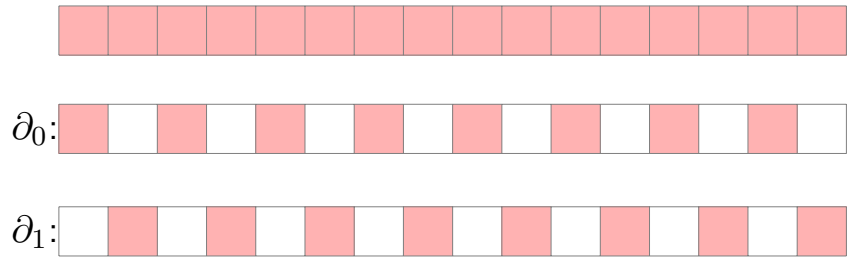

$\partial_{0} \circ \partial_{0}:$

$\partial_{1} \circ \partial_{0}:$

$\partial_{0} \circ \partial_{1}:$

$\partial_{1} \circ \partial_{1}:$

$\partial_{0}^{3}$ : 


\section{S. Eilenberg, Allouche-Shallit Thm. 6.6.2 [7]}

A sequence is $k$-automatic IFF its $k$-kernel is finite.

$\Rightarrow \mathrm{A}$ DFAO is given. The transition monoid is finite $\leq \# Q^{\# Q}$

$$
f_{u}: Q \rightarrow Q, q \mapsto q \cdot u
$$

action of $\{0, \ldots, k-1\}^{*}$ on the set of states $Q$;

- reading $\varepsilon, 1,2,10,11,12,100,101, \ldots$ provides us with the sequence $x(n)_{n \geq 0}$;

- reading $\varepsilon . u, 1 . u, 2 . u, 10 . u, 11 . u, 12 . u, 100 . u, 101 . u, \ldots$ provides us with the sequence of the $k$-kernel corresponding to $x\left(k^{|u|} n+\operatorname{val}_{k}(u)\right)_{n \geq 0}$. 

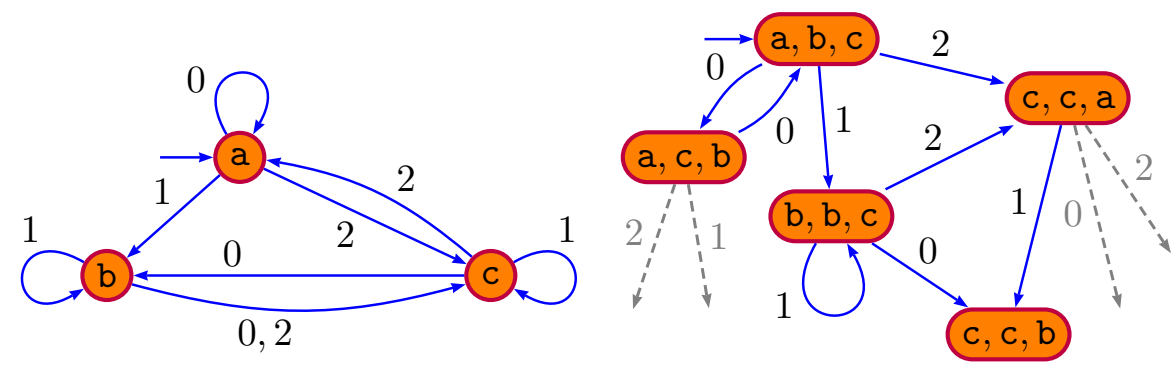

\begin{tabular}{|c|c|c|c|c|c|c|c|c|c|c|c|c|c|c|c|c|c|}
\hline & & 0 & $\neg$ & $\sim$ & 5 & $\widetilde{O}$ & 욱 & $\stackrel{\curvearrowright}{\curvearrowright}$ & N & 옹 & ণి & సิ & 공 & ָ̊ & & ิㅗ & ָָ \\
\hline$a$ & $a$ & $a$ & $b$ & $c$ & $\bar{b}$ & $c$ & $c$ & $b$ & $a$ & $c$ & $b$ & $a$ & $b$ & $c$ & $a$ & $a$ & $a$ \\
\hline$b$ & $b$ & $c$ & $b$ & $c$ & $c$ & $a$ & $c$ & $b$ & $a$ & $b$ & $a$ & $c$ & $b$ & $c$ & $a$ & $b$ & $a$ \\
\hline$c$ & $c$ & $b$ & $c$ & $a$ & $b$ & $c$ & $b$ & $a$ & $c$ & $c$ & $b$ & $a$ & $b$ & $c$ & $b$ & $a$ & $a$ \\
\hline
\end{tabular}

The 3 -kernel contains $17 \leq 3^{3}$ sequences (also depends on the coding $g$ ) 

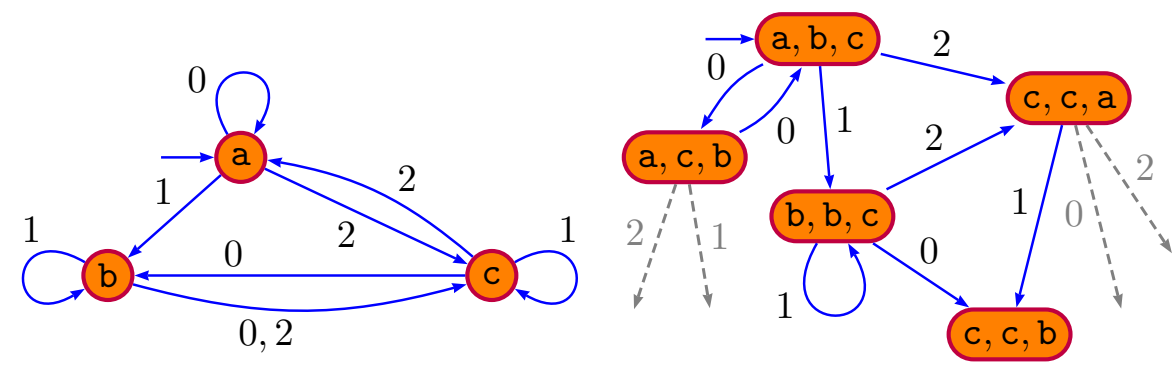

\begin{tabular}{|c|c|c|c|c|c|c|c|c|c|c|c|c|c|c|c|c|c|}
\hline & & 0 & 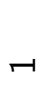 & $\sim$ & 5 & ऽ & 윽 & $\stackrel{\curvearrowright}{\sim}$ & $\approx$ & $\begin{array}{l}\text { 옹 } \\
\text { (n) }\end{array}$ & ণু & & 공 & ণิ & 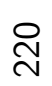 & ָָ & ָิ \\
\hline$a$ & 1 & 1 & 0 & 0 & 0 & 0 & 0 & 0 & 1 & 0 & 0 & 1 & 0 & 0 & 1 & 1 & 1 \\
\hline$b$ & 0 & 0 & 0 & 0 & 0 & 1 & 0 & 0 & 1 & 0 & 1 & 0 & 0 & 0 & 1 & 0 & 1 \\
\hline$c$ & 0 & 0 & 0 & 1 & 0 & 0 & 0 & 1 & 0 & 0 & 0 & 1 & 0 & 0 & 0 & 1 & 1 \\
\hline
\end{tabular}

The 3 -kernel contains $17 \leq 3^{3}$ sequences (also depends on the coding $g$, here 7 sequences: 011 is missing) 
Recall that the Grundy function is unbounded (e.g., game of Nim) How to generalize $k$-automatic sequences to an "infinite alphabet"?

\section{DEFINITION [16]}

$\mathbf{x} \in \mathbb{Z}^{\mathbb{N}}$ is $k$-regular if the $\mathbb{Z}$-module generated by $\operatorname{Ker}_{k}(\mathbf{x})$ is finitely generated, i.e., there exists $\mathbf{t}_{1}, \ldots, \mathbf{t}_{\ell} \in \mathbb{Z}^{\mathbb{N}}$ such that

$$
\left\langle\operatorname{Ker}_{k}(\mathbf{x})\right\rangle=\left\langle\mathbf{t}_{1}, \ldots, \mathbf{t}_{\ell}\right\rangle .
$$

$\mathbb{Z}$ is embedded in fields such as $\mathbb{Q}, \mathbb{R}$ or $\mathbb{C}$. Thus the sequences can be seen as elements of $\mathbb{Q}^{\mathbb{N}}$ which is a $\mathbb{Q}$-vector space.

The orbit of $\mathbf{x}$ under the action of the operators of decimation $\partial_{k, r}$ remains in a finite dimensional vector space. 


\section{ORIGinal DEFinition (Allouche-Shallit 1990)}

Let $R$ be a ring containing a commutative Noetherian ring $R^{\prime}$. A sequence $\mathbf{x}$ in $R^{\mathbb{N}}$ is $\left(R^{\prime}, k\right)$-regular if there exists $\mathbf{t}_{1}, \ldots, \mathbf{t}_{\ell} \in R^{\mathbb{N}}$ such that every sequence in $\operatorname{Ker}_{k}(\mathbf{x})$ is an $R^{\prime}$-linear combination of $\mathbf{t}_{1}, \ldots, \mathbf{t}_{\ell}$.

$\left\langle\operatorname{Ker}_{k}(\mathbf{x})\right\rangle$ is a submodule of a finitely generated $R^{\prime}$-module (in general, this does not imply that the submodule itself is finitely generated).

Since $R^{\prime}$ is assumed to be Noetherian, one can show that every submodule of a finitely generated $R^{\prime}$-module is finitely generated and thus $\left\langle\operatorname{Ker}_{k}(\mathbf{x})\right\rangle$ is finitely generated. 


\section{EXAMPLE}

The base- 2 sum-of-digits function $s_{2}$ gives the sequence

$$
\left(s_{2}(n)\right)_{n \geq 0}=0,1,1,2,1,2,2,3,1,2,2,3,2,3,3,4,1,2,2, \ldots .
$$

Clearly this sequence is unbounded: $s_{2}\left(2^{n}-1\right)=n$ for all $n$.

The $\mathbb{Z}$-module generated by its 2 -kernel is generated ${ }^{1}$ by the sequence $\left(s_{2}(n)\right)_{n \geq 0}$ itself and the constant sequence $(1)_{n \geq 0}$ :

$$
s_{2}(2 n)=s_{2}(n), \quad s_{2}(2 n+1)=s_{2}(n)+1
$$

\section{PROPOSITION}

Let $s$ be a sequence taking finitely many different values. Let $k \geq 2$. The sequence is $k$-automatic if and only if it is $k$-regular.

Remark: intermediate notion of $k$-synchronized sequences. [17]

${ }^{1}$ If it's unclear how to conclude from these two relations, wait a few slides, we will do it in details on another example with Nim. 
With every $k$-regular sequence $(\mathbf{x}(n))_{n \geq 0} \in \mathbb{Z}^{\mathbb{N}}$ is associated a linear representation $(\lambda, \mu, \nu)$ : there exist $r \in \mathbb{N}_{>0}, \lambda \in \mathbb{Z}^{1 \times r}$, $\nu \in \mathbb{Z}^{r \times 1}$ and a matrix-valued morphism

$\mu:\{0, \ldots, k-1\} \rightarrow \mathbb{Z}^{r \times r}$ such that

$$
\mathbf{x}(n)=\lambda \mu\left(c_{0} \cdots c_{\ell}\right) \nu
$$

for all $c_{\ell}, \ldots, c_{0} \in\{0, \ldots, k-1\}$ such that $\operatorname{val}_{k}\left(c_{\ell} \cdots c_{0}\right)=n$.

The converse holds: if there exists a linear representation associated with canonical $k$-ary expansions (take into account the technicality of leading zeros), then the sequence is $k$-regular. See, Allouche-Shallit'03 Thm. 16.2.3 [7].

\section{Corollary [16]}

The $n$th term of a $k$-regular sequence can be computed with $\left\lfloor\log _{k}(n)\right\rfloor$ matrix multiplications. 
If you are familiar with rational series:

\section{THEOREM}

A sequence $\mathbf{x}(n)$ is $k$-regular if and only if the formal series

$$
\sum_{w \in\{0, \ldots, k-1\}^{*}} \mathbf{x}\left(\operatorname{val}_{k}(w)\right) w
$$

is recognizable (in the sense of Berstel-Reutenauer [18]). 
The sequence $s_{2}=\left(s_{2}(n)\right)_{n \geq 0}$ has a (base-2) linear representation given by

$$
\lambda=\left(\begin{array}{ll}
0 & 1
\end{array}\right), \mu(0)=\left(\begin{array}{ll}
1 & 0 \\
0 & 1
\end{array}\right), \mu(1)=\left(\begin{array}{ll}
1 & 0 \\
1 & 1
\end{array}\right), \nu=\left(\begin{array}{l}
1 \\
0
\end{array}\right) .
$$

Think of $\left(s_{2}(n)\right)$ and (1) as a basis. Matrix representation of the linear operators $\partial_{2,0}$ and $\partial_{2,1}$ in that basis:

$$
\begin{gathered}
\partial_{2,0}\left(s_{2}(n)\right)=\left(s_{2}(n)\right) \text { and } \partial_{2,0}(1)=(1) ; \\
\partial_{2,1}\left(s_{2}(n)\right)=\left(s_{2}(n)\right)+(1) \text { and } \partial_{2,1}(1)=(1) .
\end{gathered}
$$




\section{SOME APPLICATIONS OF ITERATED MORPHISMS}

- overlaps are avoidable over a 2-letter alphabet $[19,20,21]$ abbabaabbaababbabaababbaabbabaab ...

$$
a \mapsto a b, \quad b \mapsto b a ;
$$

- cubes are avoidable over a 3 -letter alphabet

$$
\begin{gathered}
a b b|a b| a|a b b| a|a b| a b b|a b| a|a b| a b b|a| a b b|a b| a \mid a b \cdots \\
321312321231321 \ldots \\
3 \mapsto 321, \quad 2 \mapsto 31, \quad 1 \mapsto 2 .
\end{gathered}
$$




\section{Keränen (ICALP'1992) avoiding abelian squares [22]}

$a \mapsto a b c a c d c b c d c a d c d b d a b a c a b a d b a b c b d b c b a c b c d c a c b a b d$ abacadcbcdcacdbcbacbcdcacdcbdcdadbdcbca;

$b \mapsto b c d b d a d c d a d b a d a c a b c b d b c b a c b c d c a c d c b d c d a d b d c b c a$ bcbdbadcdadbdacdcbdcdadbdadcadabacadcdb;

$c \mapsto c d a c a b a d a b a c b a b d b c d c a c d c b d c d a d b d a d c a d a b a c a d c d b$ cdcacbadabacabdadcadabacabadbabcbdbadac;

$d \mapsto d a b d b c b a b c b d c b c a c d a d b d a d c a d a b a c a b a d b a b c b d b a d a c$ dadbdcbabcbdbcabadbabcbdbcbacbcdcacbabd; 
J. Cassaigne, J. D. Currie, L. Schaeffer, J. Shallit, Avoiding Three Consecutive Blocks of the Same Size and Same Sum [23]

$$
\begin{gathered}
\varphi: 0 \mapsto 03,1 \mapsto 43,3 \mapsto 1,4 \mapsto 01 \\
\varphi^{\omega}(0)=031430110343430310110110314303434303434 \cdots
\end{gathered}
$$

has no additive cube, e.g., 041340. 
Prouhet's problem (1851) - Tarry - Escott [24, 25, 26] Mémoire sur quelques relations entre les puissances de nombres

Partition $\left\{0, \ldots, 2^{n}-1\right\}$ such that

$$
\begin{gathered}
\left\{0, \ldots, 2^{n}-1\right\}=\left\{a_{1}, \ldots, a_{2^{n-1}}\right\} \cup\left\{b_{1}, \ldots, b_{2^{n-1}}\right\} \\
\sum_{j=1}^{2^{n-1}} a_{j}=\sum_{j=1}^{2^{n-1}} b_{j}, \sum_{j=1}^{2^{n-1}} a_{j}^{2}=\sum_{j=1}^{2^{n-1}} b_{j}^{2}, \ldots, \sum_{j=1}^{2^{n-1}} a_{j}^{n-1}=\sum_{j=1}^{2^{n-1}} b_{j}^{n-1} \\
\prod_{i=0}^{\infty}\left(1-X^{2^{i}}\right)=\sum_{j=0}^{\infty} t_{j} X^{j}
\end{gathered}
$$




\section{EXTENSION TO A MULTIDIMENSIONAL SETTING}

There are natural extensions [10]

- Image under a coding of a fixed point of a $k$-uniform morphism;

$$
a \mapsto \begin{array}{|c|c|}
\hline a & b \\
\hline b & a \\
\hline
\end{array} \quad b \mapsto \begin{array}{|l|l|}
\hline a & b \\
\hline a & b \\
\hline
\end{array}
$$

- Sequence of outputs of a DFAO fed with base- $k$ expansions;

$$
q \stackrel{\left(\begin{array}{l}
0 \\
1
\end{array}\right)}{\longrightarrow} q^{\prime} \quad \text { padding shorter expansions with } 0
$$

- Sets of indices defined by a first order formula in $\left\langle\mathbb{N},+, V_{k}\right\rangle$;

$$
\psi_{a}(x, y) \equiv \cdots
$$

- Finiteness of the $k$-kernel.

Remark: one could define $(k, \ell)$-automatic sequences (see, again, Allouche-Shallit 2003, [7, Chap. 14]). 

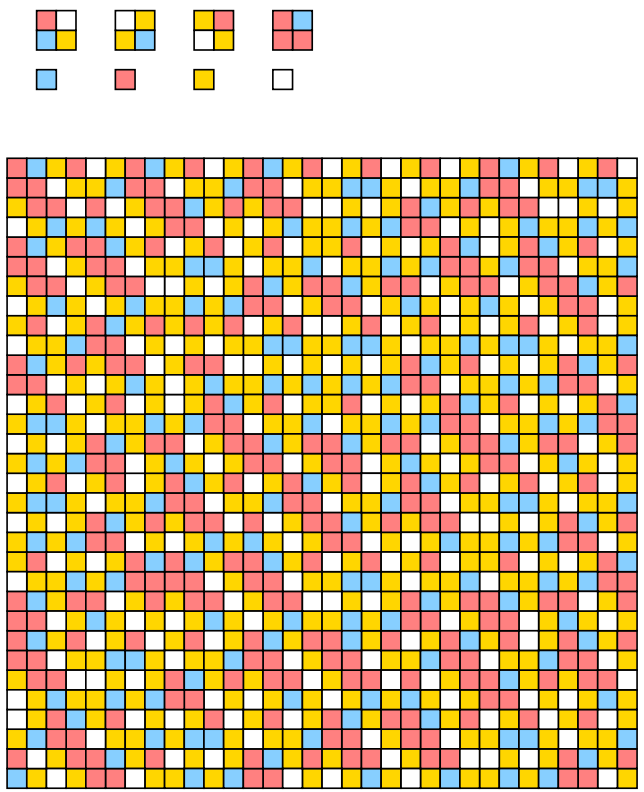

O. Salon, Suites automatiques à multi-indices, Séminaire de théorie des nombres, Bordeaux, 1986-1987, exposé 4. [44] 


\section{Tracking the past}

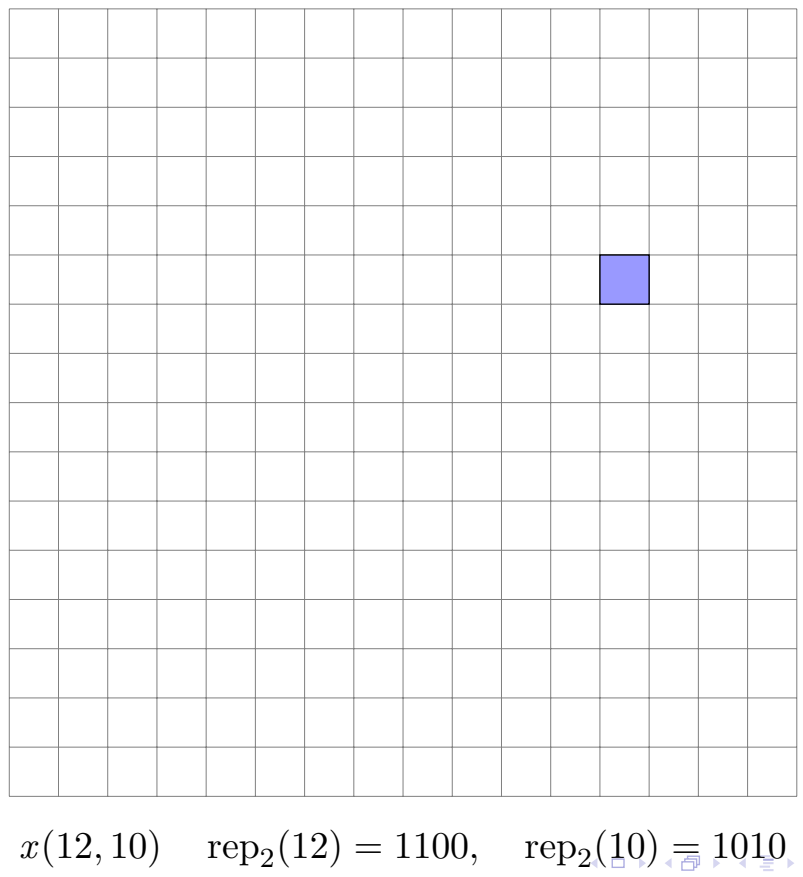




\section{Tracking the past}

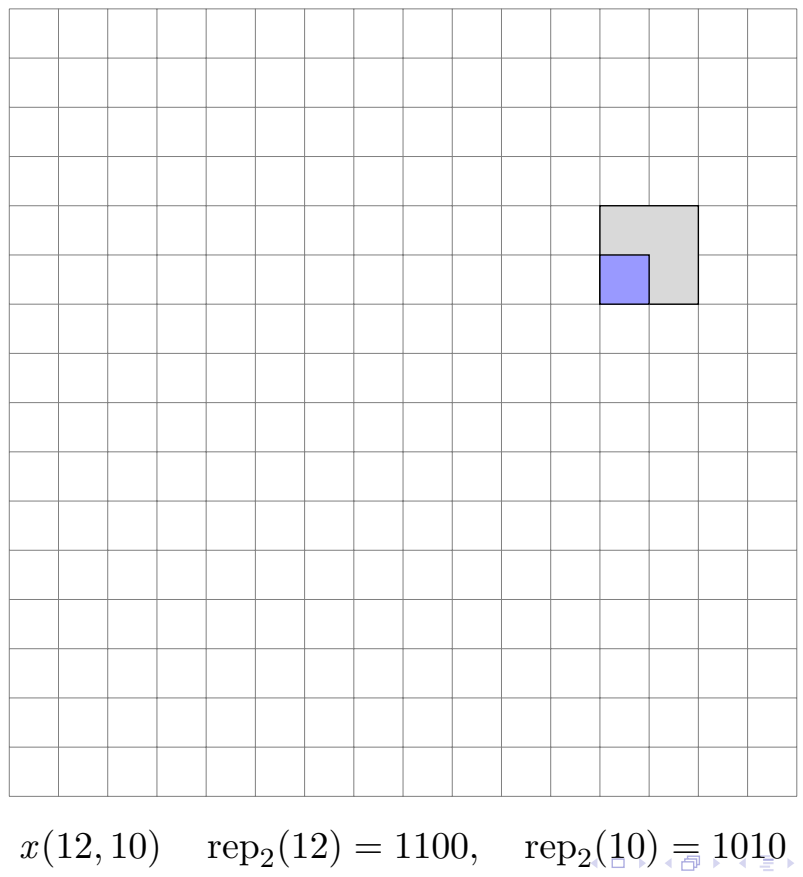




\section{Tracking the past}

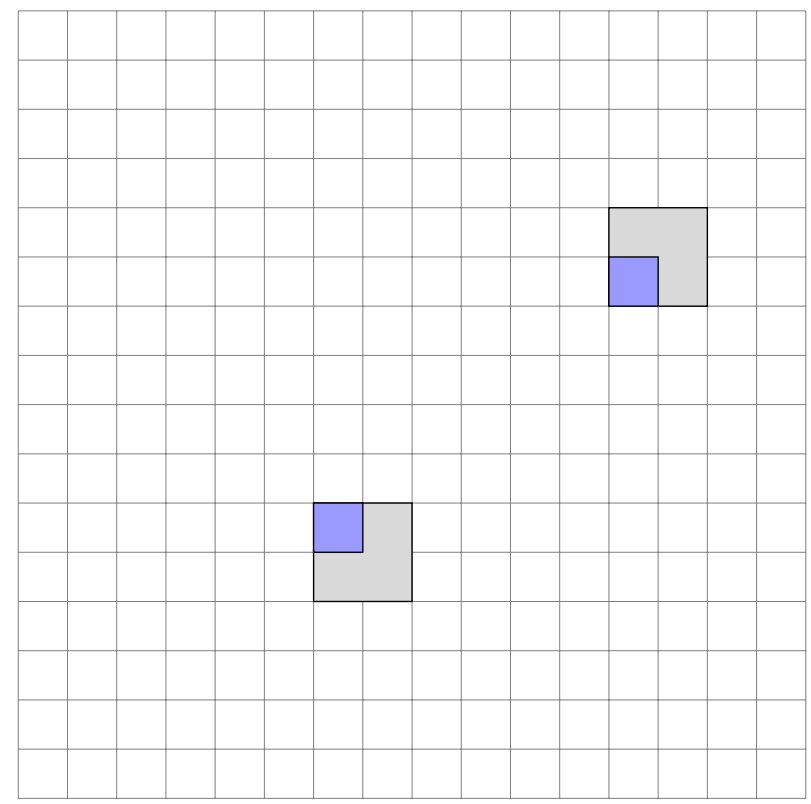

$x(6,5) \rightarrow x(12,10) \quad \operatorname{rep}_{2}(6)=110, \quad \operatorname{rep}_{2}(5)=101$ 


\section{Tracking the past}

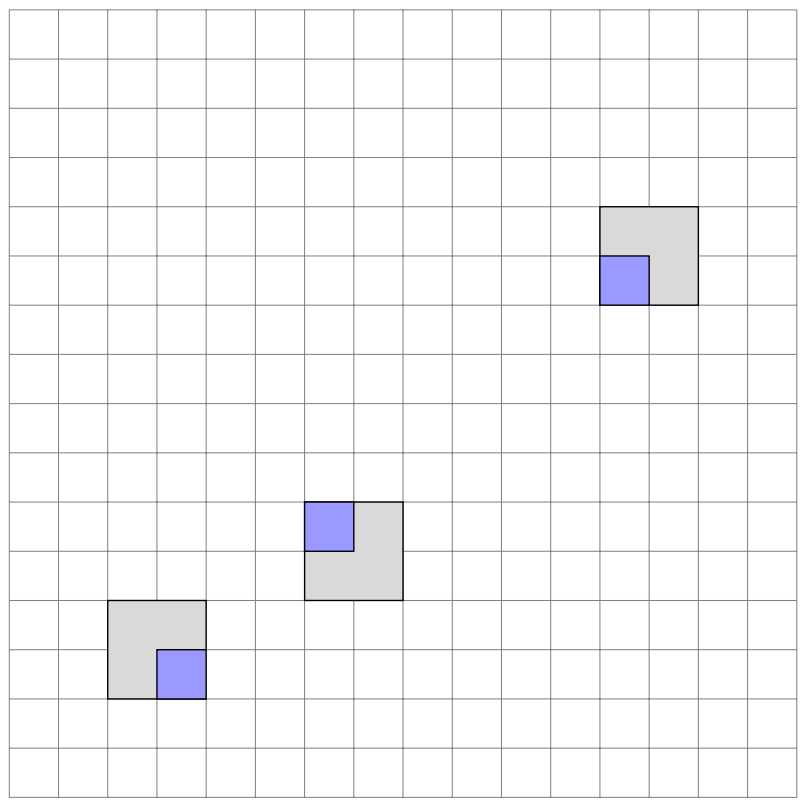

$x(3,2) \rightarrow x(6,5) \rightarrow x(12,10) \quad \operatorname{rep}_{2}(3)=11, \quad \operatorname{rep}_{2}(2)=10$ 


\section{Tracking the past}

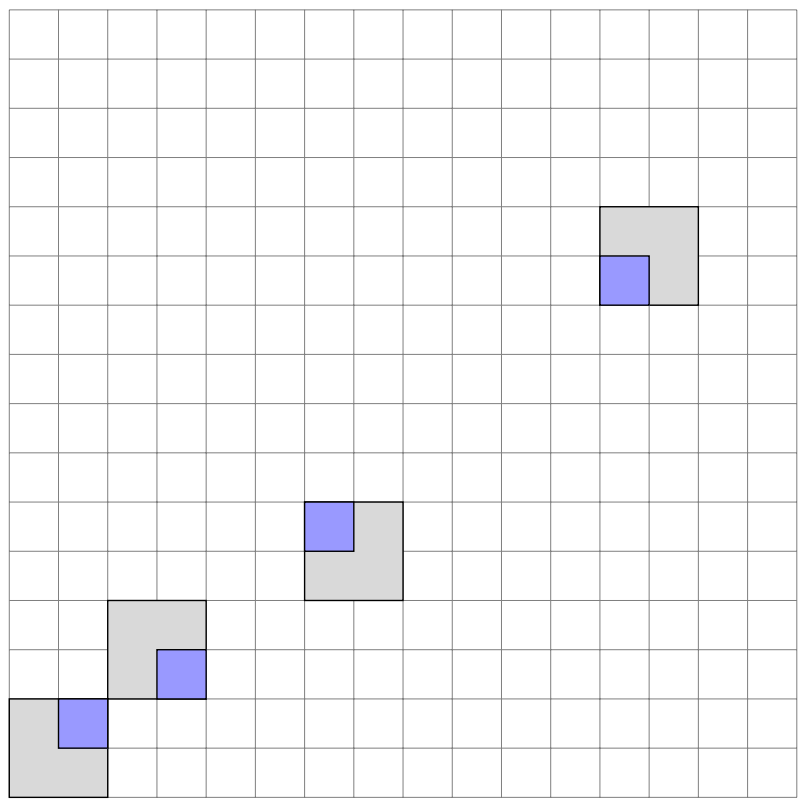

$x(1,1) \rightarrow x(3,2) \rightarrow x(6,5) \rightarrow x(12,10) \quad \operatorname{rep}_{2}(3) \equiv 1, \quad \operatorname{rep}_{2}(2)=1$ 
Definition of the $k$-kernel in a multidimensional setting

\section{DEFINITION}

Consider a bi-dimensional sequence $\mathbf{x}=(x(m, n))_{m, n \geq 0}$. It is a set of bi-dimensional subsequences:

$$
\operatorname{Ker}_{k}(\mathbf{x})=\left\{\left(x\left(k^{i} m+r, k^{i} n+s\right)\right)_{m, n \geq 0} \mid i \geq 0,0 \leq r, s<k^{i}\right\} .
$$

This corresponds to selecting the suffixes

$$
\left(0^{i-p} r_{p} \cdots r_{1}, 0^{i-q} s_{q} \cdots s_{1}\right)
$$

where $\operatorname{rep}_{k}(r)=r_{p} \cdots r_{1}$ and $\operatorname{rep}_{k}(s)=s_{q} \cdots s_{1}$. 
Some of these subsequences $(0,0)$

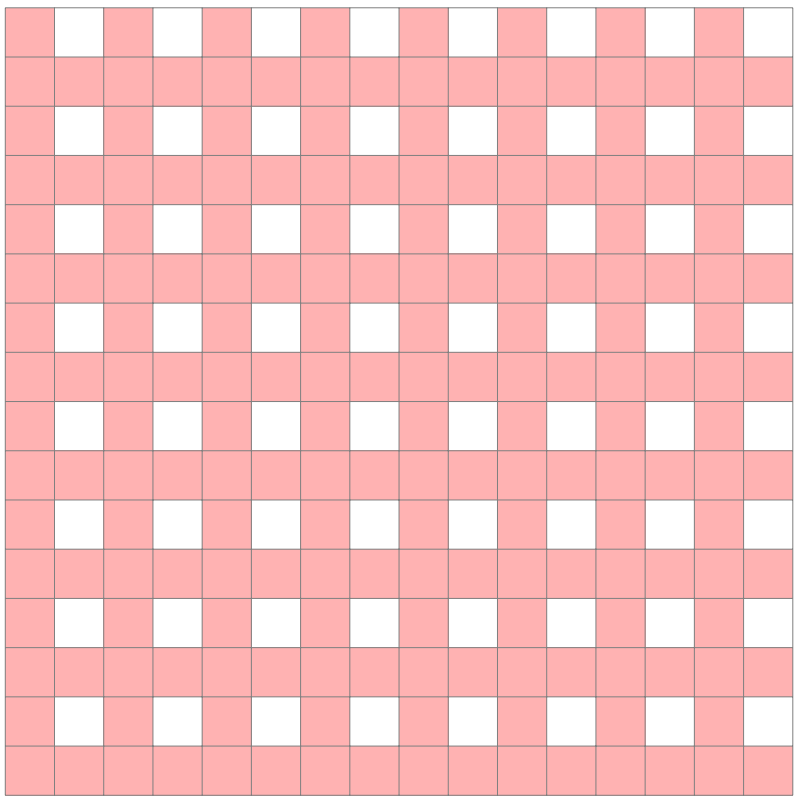


Some of these subsequences $(1,0)$

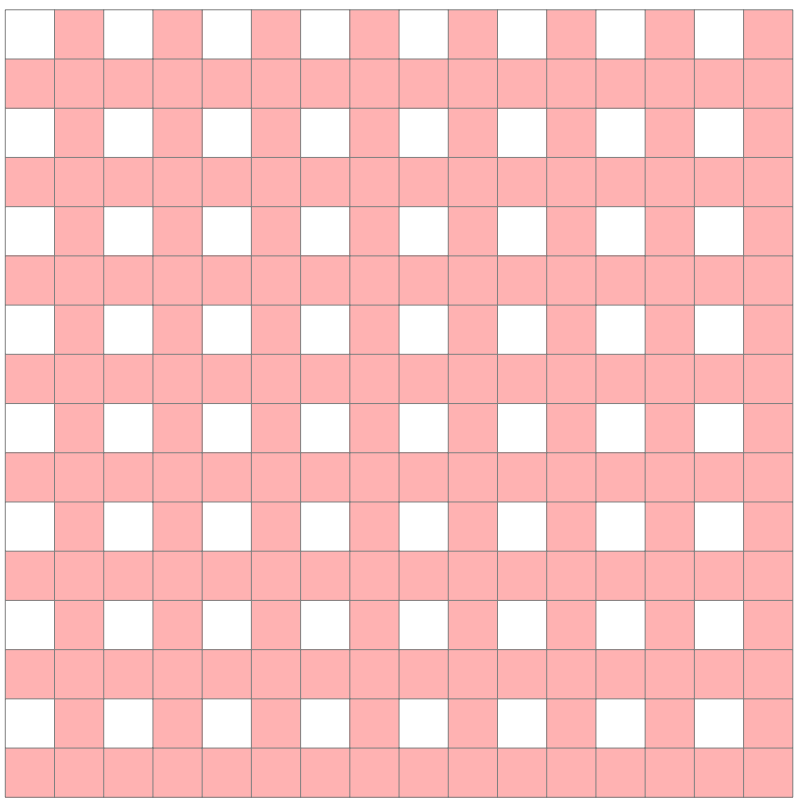


Some of these subsequences $(0,1)$

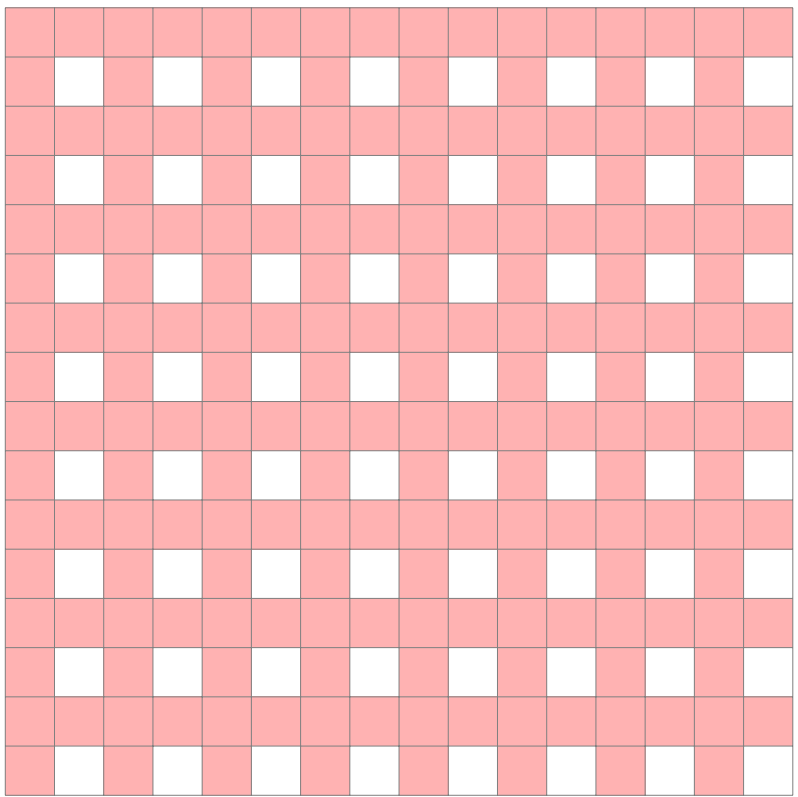


Some of these subsequences $(1,1)$

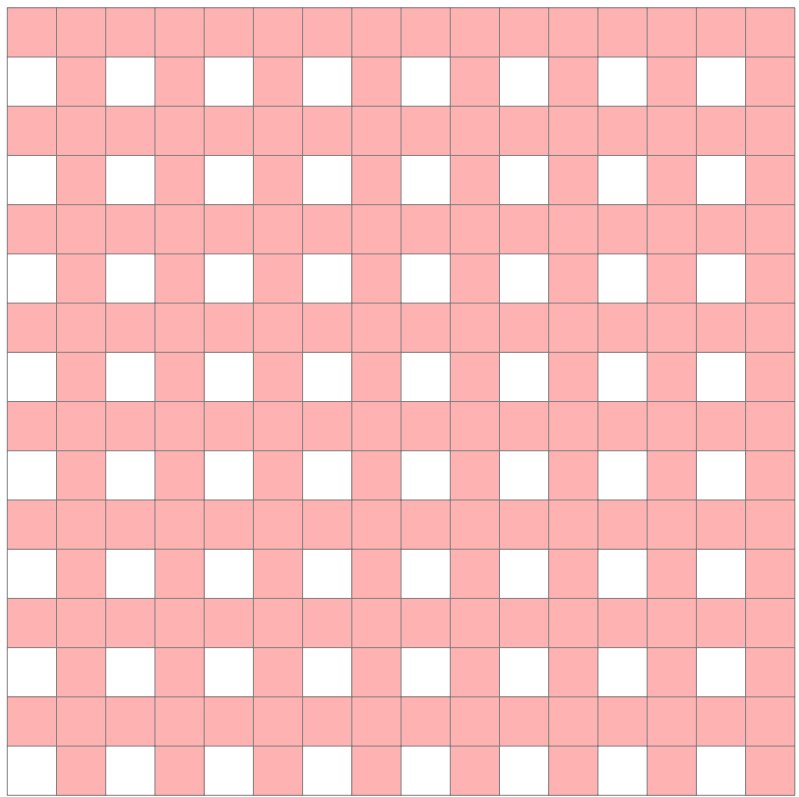


Some of these subsequences $(00,00)$

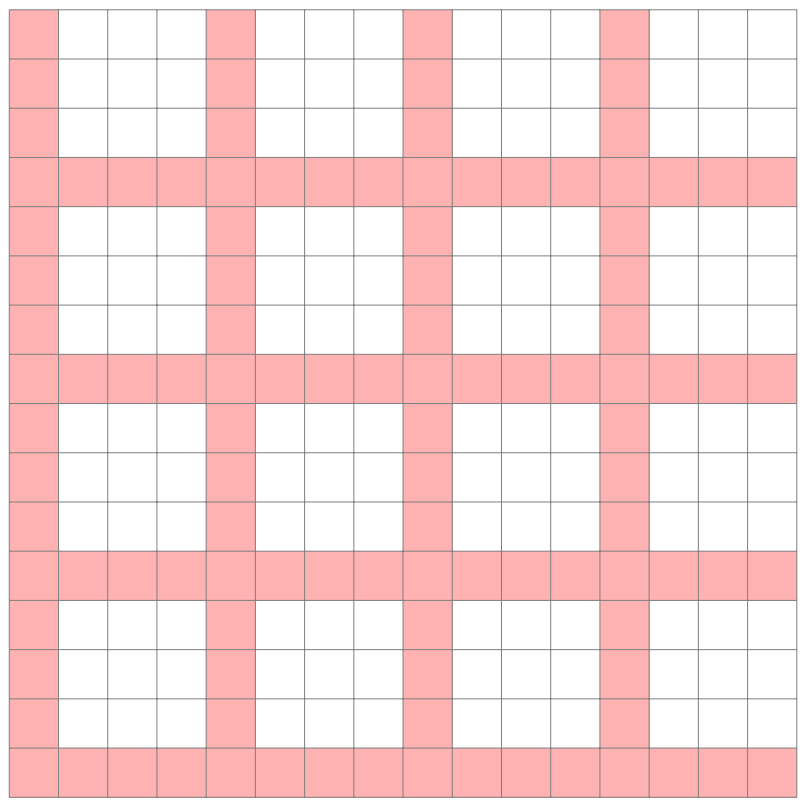


Some of these subsequences $(01,00)$

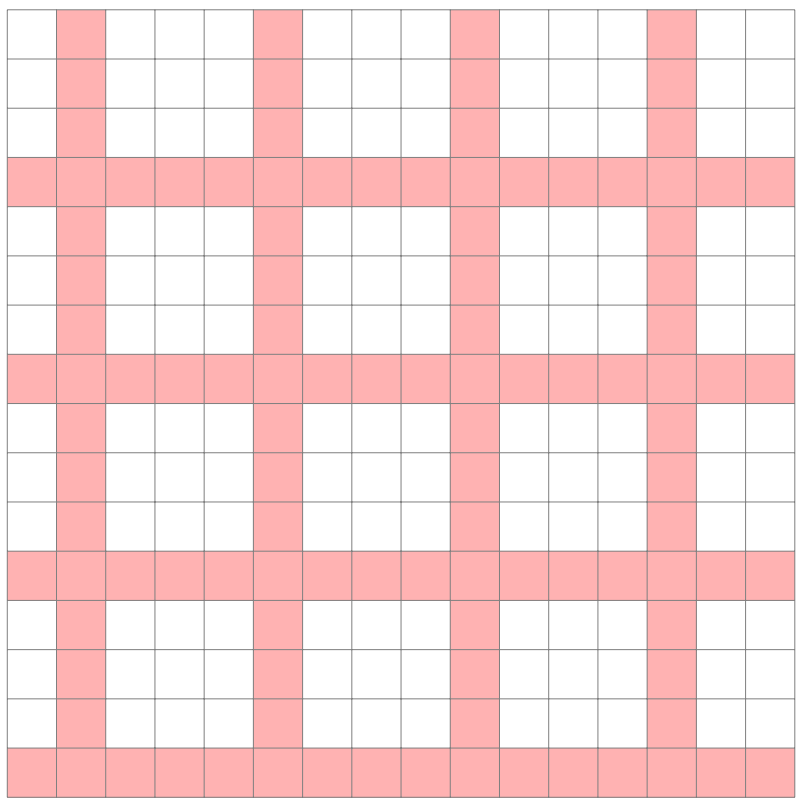


Some of these subsequences $(10,00)$

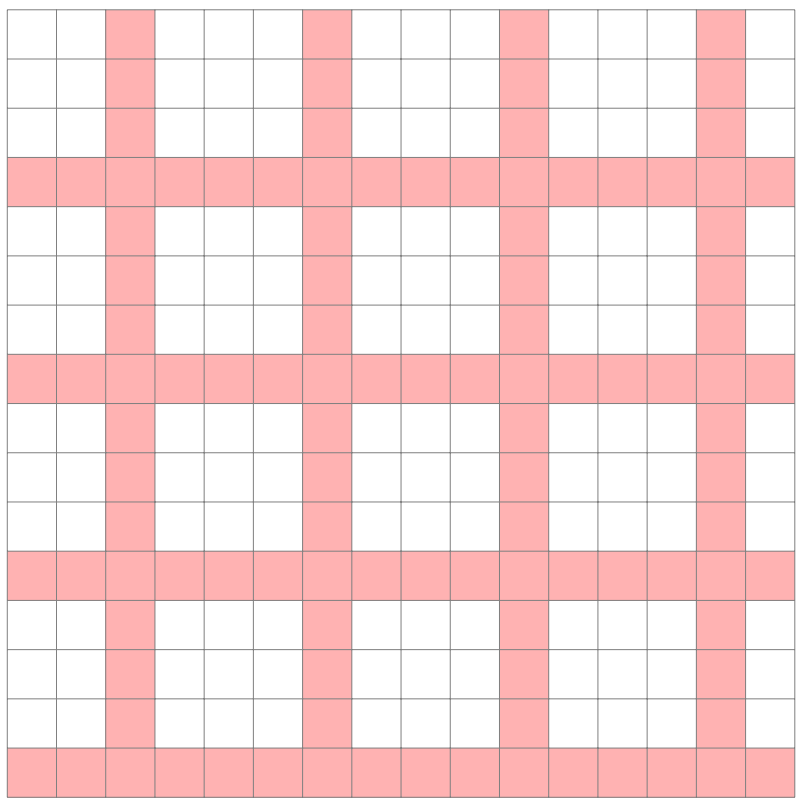


$\rightsquigarrow$ We can define multidimensional $k$-regular sequences.

The $\mathbb{Z}$-module generated by $\operatorname{Ker}_{k}(\mathbf{x})$ is finitely generated.

\section{Proposition (ExERCISE)}

For the game of $\operatorname{Nim},\left(\mathcal{G}_{N}(m, n)\right)_{m, n \geq 0}$ is 2-regular.

Proof. We have

$$
\begin{aligned}
& \mathcal{G}_{N}(2 m, 2 n)=\quad 2 m \oplus 2 n \quad=2 \mathcal{G}_{N}(m, n) \\
& \mathcal{G}_{N}(2 m+1,2 n)=(2 m+1) \oplus 2 n \quad=2 \mathcal{G}_{N}(m, n)+1 \\
& \mathcal{G}_{N}(2 m, 2 n+1)=2 m \oplus(2 n+1) \quad=2 \mathcal{G}_{N}(m, n)+1 \\
& \mathcal{G}_{N}(2 m+1,2 n+1)=(2 m+1) \oplus(2 n+1)=2 \mathcal{G}_{N}(m, n)
\end{aligned}
$$

thus the 2-kernel is generated by $\left(\mathcal{G}_{N}(m, n)\right)_{m, n \geq 0}$ and the constant sequence (1). 
Is that clear for any element of the 2-kernel?

Can $\left(\mathcal{G}_{N}(8 m+5,8 n+2)\right)_{m, n \geq 0}$ be expressed as a $\mathbb{Z}$-linear combination of these two sequences?

$$
\begin{aligned}
\mathcal{G}_{N}(8 m+5,8 n+2) & =\mathcal{G}_{N}(2(4 m+2)+1,2(4 n+1)) \\
& =2 \mathcal{G}_{N}(4 m+2,4 n+1)+1 \\
& =2 \mathcal{G}_{N}(2(2 m+1), 2.2 n+1)+1 \\
& =2\left[2 \mathcal{G}_{N}(2 m+1,2 n)+1\right]+1 \\
& =4 \mathcal{G}_{N}(2 m+1,2 n)+3 \\
& =4\left[2 \mathcal{G}_{N}(m, n)+1\right]+3 \\
& =8 \mathcal{G}_{N}(m, n)+7 .
\end{aligned}
$$


Meaning of these relations within the table:

$$
\begin{array}{cccccccccc}
9 & 8 & 11 & 10 & 13 & 12 & 15 & 14 & 1 & 0 \\
8 & 9 & 10 & 11 & 12 & 13 & 14 & 15 & 0 & 1 \\
\hline 7 & 6 & 5 & 4 & 3 & 2 & 1 & 0 & 15 & 14 \\
6 & 7 & 4 & 5 & 2 & 3 & 0 & 1 & 14 & 15 \\
5 & 4 & 7 & 6 & 1 & 0 & 3 & 2 & 13 & 12 \\
4 & 5 & 6 & 7 & 0 & 1 & 2 & 3 & 12 & 13 \\
3 & 2 & 1 & 0 & 7 & 6 & 5 & 4 & 11 & 10 \\
2 & 3 & 0 & 1 & 6 & 7 & 4 & 5 & 10 & 11 \\
1 & 0 & 3 & 2 & 5 & 4 & 7 & 6 & 9 & 8 \\
0 & 1 & 2 & 3 & 4 & 5 & 6 & 7 & 8 & 9
\end{array}
$$

First few values of $\mathcal{G}_{N}(m, n)$.

$$
\mathcal{G}_{N}(m, n) \mapsto \begin{array}{|c|c|}
\hline 2 \mathcal{G}_{N}(m, n)+1 & 2 \mathcal{G}_{N}(m, n) \\
\hline 2 \mathcal{G}_{N}(m, n) & 2 \mathcal{G}_{N}(m, n)+1 \\
\hline
\end{array}
$$


For the game of Wythoff, first few values of $(x, y) \mapsto \mathcal{G}_{W}(x, y)$

\begin{tabular}{c|ccccccccccc}
$\vdots$ & & & & & & & & & & & \\
9 & 9 & 10 & 11 & 12 & 8 & 7 & 13 & 14 & 15 & 16 & \\
8 & 8 & 6 & 7 & 10 & 1 & 1 & 5 & 3 & 4 & 15 & \\
7 & 7 & 8 & 6 & 9 & 0 & 1 & 4 & 5 & 3 & 14 & \\
6 & 6 & 7 & 8 & 1 & 9 & 10 & 3 & 4 & 5 & 13 & \\
5 & 5 & 3 & 4 & 0 & 6 & 8 & 10 & 1 & 2 & 7 & \\
4 & 4 & 5 & 3 & 2 & 7 & 6 & 9 & 0 & 1 & 8 & \\
3 & 3 & 4 & 5 & 6 & 2 & 0 & 1 & 9 & 10 & 12 & \\
2 & 2 & 0 & 1 & 5 & 3 & 4 & 8 & 6 & 7 & 11 & \\
1 & 1 & 2 & 0 & 4 & 5 & 3 & 7 & 8 & 6 & 10 & \\
0 & 0 & 1 & 2 & 3 & 4 & 5 & 6 & 7 & 8 & 9 & $\cdots$ \\
\hline & 0 & 1 & 2 & 3 & 4 & 5 & 6 & 7 & 8 & 9 & $\cdots$
\end{tabular}


Not so many results are known

- U. Blass, A.S. Fraenkel, The Sprague-Grundy function for Wythoff's game. Theoret. Comput. Sci. 75 (1990), no. 3, 311-333. [27]

- Y. Jiao, On the Sprague-Grundy values of the $\mathcal{F}$-Wythoff game. Electron. J. Combin. 20 (2013). [28]

- A. Gu, Sprague-Grundy values of the $\mathcal{R}$-Wythoff game. Electron. J. Combin. 22 (2015). [29]

- M. Weinstein, Invariance of the Sprague-Grundy function for variants of Wythoff's game. Integers 16 (2016). [30]

It's challenging, we quote the book [1, A. Siegel, p. 200]:

"No general formula is known for computing arbitrary $\mathcal{G}$-values of WYTHOFF. In general, they appear chaotic, though they exhibit a striking fractal-like pattern ... Despite this apparent chaos, the $\mathcal{G}$-values nonetheless have a high degree of geometric regularity." 


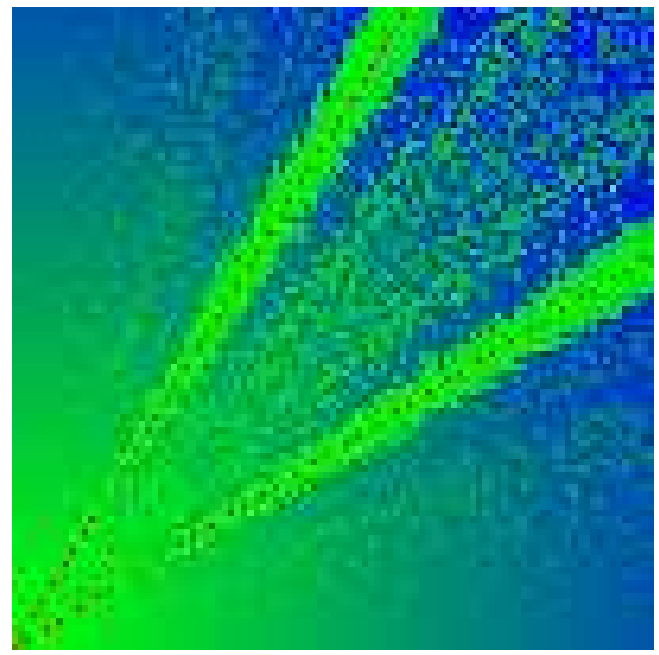

$\mathcal{G}_{W}(m, n), m, n \leq 100$ 
Defining the MeX function for a list

$\operatorname{mex}[\{\}]=0$;

$\operatorname{mex}\left[1_{-}\right]:=\operatorname{Min}[$ Complement $[\mathrm{Table}[i,\{i, 0, \operatorname{Max}[1]+1\}], 1]]$

List the available pairs of positions

$\ln [19]:=\operatorname{pos}\left[x_{-} / ; x>0, y_{-} / ; y>0\right]:=\operatorname{Union}[\mathrm{Table}[\{i, y\},\{i, 0, x-1\}]$,

Table $[\{x, i\},\{i, 0, y-1\}]$, Table $[\{x-i, y-i\},\{i, 1, \operatorname{Min}[\{x, y\}]\}]]$;

$\operatorname{pos}\left[0, y_{-} / ; y>0\right]:=$ Table $[\{0, i\},\{i, 0, y-1\}]$;

pos $\left[x_{-} / ; x>0,0\right]:=$ Table $[\{i, 0\},\{i, 0, x-1\}]$;

Compute the Sprage-Grundy function for Wythoff's game

$\ln [28]:=\mathrm{W}[\{0,0\}]=0$;

$\mathrm{w}\left[\left\{x_{-}, y_{-}\right\}\right]:=\mathrm{w}[\{x, y\}]=\operatorname{mex}[\operatorname{Map}[\mathrm{w}[\#] \&, \operatorname{pos}[x, y]]]$

$\ln [50]:=$ Timing [

$t=\operatorname{Table}[\mathrm{w}[\{i, j\}],\{i, 0,100\},\{j, 0,100\}] ;]$

Out[50]=

$\{2.03782, \mathrm{Nu} 11\}$

Give nice RGB colors, 0 is mapped to Red, other values from Green to Blue

$\operatorname{In}[51]:=\operatorname{Graphics}[\operatorname{Raster}[\operatorname{Map}[\operatorname{If}[\#>0,\{0, \operatorname{Max}[t]-\#, \#\} / \operatorname{Max}[t],\{1,0,0\}] \&, t,\{2\}]]]$ 
Some results from Blass and Fraenkel [27]:

- On every parallel to the main diagonal, $\mathcal{G}_{W}(n, n+j)$ takes every possible value.

- Points with Grundy value 1 are "close" to those with value 0 .

- Recursive algorithm to determine the points with Grundy value 1 . 
Additive periodicity of the rows of $\left(\mathcal{G}_{W}(m, n)\right)$

\section{DEFINITION}

A sequence $\left(a_{j}\right)_{j \geq 0}$ is additively periodic if

$$
\exists p, q, \forall j \geq q: a_{j+p}=a_{j}+p .
$$

- A. Dress, A. Flammenkamp and N. Pink, Additive periodicity of the Sprague-Grundy function of certain Nim games, Adv. in Appl. Math. 22, 249-270 (1999). [31]

- H. A. Landman, A simple FSM²-based proof of the additive periodicity of the Sprague-Grundy function of Wythoff's game, More Games of No Chance, 2002. [32] 
As an example, the row $\mathcal{G}_{W}(5, n)$ is such that for all $n \geq 27, \mathcal{G}_{W}(5, n+24)=\mathcal{G}_{W}(5, n)+24$

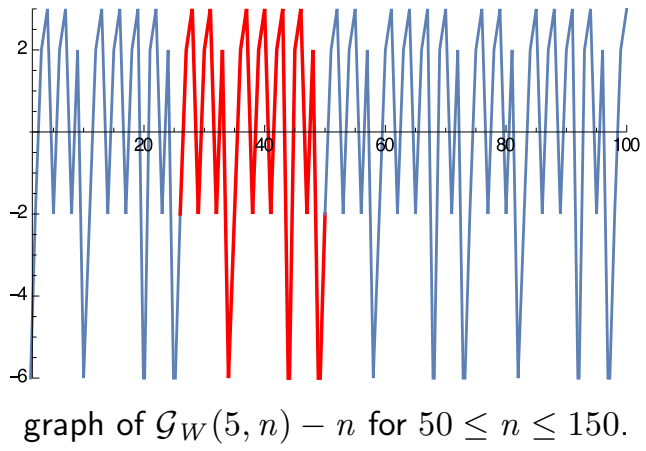


Observe that the Fibonacci word is coding the $\mathcal{P}$-positions:

We have a Beatty sequence $\frac{1}{\varphi}+\frac{1}{\varphi^{2}}=1$,

$(\lfloor n \varphi\rfloor)_{n \geq 1}$ and $\left(\left\lfloor n \varphi^{2}\right\rfloor\right)_{n \geq 1}$ make a partition of $\mathbb{N}_{>0}$ [33]

$$
1,2,3,4,5,6,7,8,9,10,11,12, \cdots
$$

This corresponds to the Sturmian (Fibonacci) word: $\mathbf{f}=a b a a b a b a a b \cdots=2122121221 \cdots$

$\mathbf{f}_{n}=\lfloor\varphi(n+2)\rfloor-\lfloor\varphi(n+1)\rfloor, \quad \forall n \geq 0$.

A word is Sturmian iff it is irrational mechanical [34].

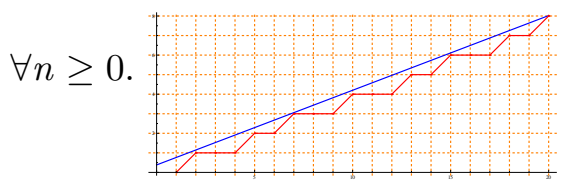

\section{PROPOSITION}

The $n$th $\mathcal{P}$-position of Wythoff's game is given by the positions (starting with 1) of the $n$th $a$ and $n$th $b$ in $\mathbf{f}$. 
Link with (Zeckendorf) numeration system [35]:

The Fibonacci word is the fixed point of $a \mapsto a b, b \mapsto a$.

What could be the analogue to

- Image under a coding of a fixed point of a $k$-uniform morphism;

- Sequence of outputs of a DFAO fed with base- $k$ expansions;

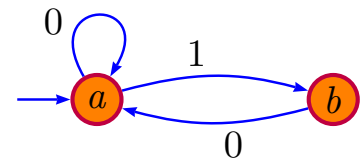

$\varepsilon, 1,10,100,101,1000,1001,1010,10000,10001,10010,10100, \ldots$

This is exactly the language of (greedy) Fibonacci representations $\operatorname{rep}_{F}(\mathbb{N})$. We have a "Fibonacci-automatic sequence". 
We have a general result (indexing starts with 0 ):

\section{General THEOREM "MORPHiC $\Rightarrow$ AUTOMATIC" [40]}

Let $A$ be an ordered alphabet. Let $\mathbf{w} \in A^{\mathbb{N}}$ be an infinite word, fixed point $f^{\omega}(a)$ of a morphism $f: A^{*} \rightarrow A^{*}$.

- associate with $f$ a DFA $\mathcal{M}$ over the alphabet $\{0, \ldots, \max |f(b)|-1\}$;

- $A$ is the set of states;

- the initial state is $a$, all states are final;

- if $f(b)=c_{0} \cdots c_{m}$, then $b \stackrel{j}{\longrightarrow} c_{j}, j \leq m$;

- consider the language $L$ accepted by $\mathcal{M}$ except words starting with 0 ;

- genealogically order $L: L=\left\{w_{0}<w_{1}<w_{2}<\cdots\right\}$.

The $n$th symbol of $\mathbf{w}, n \geq 0$, is $\mathcal{M} \cdot w_{n}$.

$\rightsquigarrow$ The ordered language $L$ plays the rôle of the base- $k$ numeration system. We have a " $L$-automatic sequence". 
Many links with non-standard numeration systems

J. Shallit (1988), J.-P. Allouche, E. Cateland, et al. (1997), J.-P.

Allouche, K. Scheicher, R. Tichy (2000), M. R.

$(2000), \ldots[36,37,38,39,40]$ 
Starting with index 0: $f_{0} f_{1} f_{2} \cdots=a b a a b \cdots$

$$
f_{j}=a \text { iff } \operatorname{rep}_{F}(j) \text { ends with } 0 ; f_{j}=b \text { iff } \operatorname{rep}_{F}(j) \text { ends with } 1 .
$$

We can still "keep track of the past": since $a \mapsto a b, b \mapsto a$, the $n$th symbol $b$ comes from the $n$th a in $\mathbf{f}$.

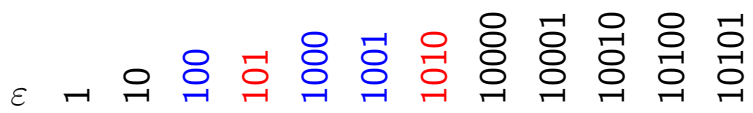

$$
\begin{aligned}
& \begin{array}{llllllllllllll}
a & b & a & a & b & a & b & a & a & b & a & a & b & \cdots
\end{array} \\
& f_{j}=a \text { and } \operatorname{rep}_{F}(j)=u 0 \\
& \text { iff } \\
& f_{\ell-1}=a, f_{\ell}=b \text { with } \ell=\operatorname{val}_{F}(u 01) . \\
& f_{j}=b \text { and } \operatorname{rep}_{F}(j)=v 1 \text { iff } f_{\ell}=a \text { with } \ell=\operatorname{val}_{F}(v 10) .
\end{aligned}
$$


Putting together the four previous slides:

\section{PROPOSITION}

The $n$th $\mathcal{P}$-position of Wythoff's game is given by the positions (starting with 1 ) of the $n$th $a$ and $n$th $b$ in $\mathbf{f}$.

$(x, y)$, with $x<y$, is a $\mathcal{P}$-position iff

$$
\left(\operatorname{rep}_{F}(x-1), \operatorname{rep}_{F}(y-1)\right)=(u 0, u 01)
$$

where $u$ is a greedy (valid) $F$-representation.

What can be said about the form of $\operatorname{rep}_{F}(x)$ and $\operatorname{rep}_{F}(y)$ ?

$$
\begin{gathered}
\operatorname{rep}_{F}(x)=\operatorname{rep}_{F}\left(\operatorname{val}_{F}(u 0)+1\right) \\
\operatorname{rep}_{F}(y)=\operatorname{rep}_{F}\left(\operatorname{val}_{F}(u 01)+1\right)
\end{gathered}
$$


- First case ${ }^{3}: u=u^{\prime} 0$

$$
\begin{gathered}
\operatorname{rep}_{F}(x)=\operatorname{rep}_{F}\left(\operatorname{val}_{F}\left(u^{\prime} 00\right)+1\right)=u^{\prime} 01 \text { ends with no zero } \\
\operatorname{rep}_{F}(y)=\operatorname{rep}_{F}\left(\operatorname{val}_{F}\left(u^{\prime} 001\right)+1\right)=u^{\prime} 010 \text { shift by one zero }
\end{gathered}
$$

- Second case : $u=u^{\prime} 1$ ( $u^{\prime}$ ends with 0$)$

$$
\begin{gathered}
\operatorname{rep}_{F}(x)=\operatorname{rep}_{F}\left(\operatorname{val}_{F}\left(u^{\prime} 10\right)+1\right)=u^{\prime} 11 \\
\operatorname{rep}_{F}(y)=\operatorname{rep}_{F}\left(\operatorname{val}_{F}\left(u^{\prime} 101\right)+1\right)=u^{\prime} 110
\end{gathered}
$$

Normalize $u^{\prime} 11 / u^{\prime} 110$ or compute the successor of $u^{\prime} 10 / u^{\prime} 101$ Transducer ( $R$ to $L$ ) computing the successor, see [41, Frougny'97]. 


$$
\begin{aligned}
& 0 \mid \text {. } \\
& 0|0,1| 1 \\
& \text { (5) } \\
& s \stackrel{\left(\begin{array}{l}
x 1 \\
x 1
\end{array}\right)}{\longleftarrow} s \stackrel{\left(\begin{array}{l}
0 \\
01
\end{array}\right)}{\longleftarrow} r\left[\stackrel{\left(\begin{array}{l}
0 \\
0
\end{array}\right)}{\longleftarrow} q \stackrel{\left(\begin{array}{l}
1 \\
0
\end{array}\right)}{\longleftarrow} r\right] \stackrel{\left(\begin{array}{l}
0 \\
0
\end{array}\right)}{\longleftarrow} q \stackrel{\left(\begin{array}{l}
1 \\
0
\end{array}\right)}{\longleftarrow} r \stackrel{\left(\begin{array}{l}
0 \\
\longleftarrow
\end{array}\right)}{\longleftarrow} p \text { :from right } \\
& \underbrace{x 10(01)^{n} 0}_{u^{\prime}} 10 \rightarrow x 101(00)^{n} 00 \quad 2 n+2 \text { zeroes, } n \geq 0
\end{aligned}
$$

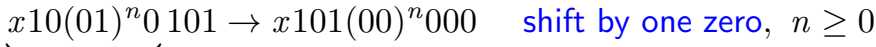

$$
\begin{aligned}
& \underbrace{}_{u^{\prime}} \\
& \underbrace{1(01)^{n} 0}_{u^{\prime}} 10 \rightarrow 100(00)^{n} 00 \quad 2 n+4 \text { zeroes, } n \geq 0 \\
& 1(01)^{n} 0101 \rightarrow 100(00)^{n} 000 \quad \text { shift by one zero, } n \geq 0
\end{aligned}
$$




\section{Corollary (A. S. Fraenkel, $1982[42]$ )}

$(x, y)$, with $x<y$, is a $\mathcal{P}$-position iff $\operatorname{rep}_{F}(x)$ ends with an even number of zeroes and $\operatorname{rep}_{F}(y)=\operatorname{rep}_{F}(x) 0$. 


\section{SHAPE-SYMMETRY}

Question: What can be said about the (morphic) structure of the $\mathcal{P}$-positions of Wythoff's 㷔 game? [43]

$$
\left(P_{i, j}\right)_{i, j \geq 0}=\mid \begin{array}{llllllllllll}
\vdots & & & & & & & & & & & \\
0 & 0 & 0 & 0 & 0 & 0 & \mathbf{1} & 0 & 0 & 0 & 0 & \\
0 & 0 & 0 & 0 & 0 & 0 & 0 & 0 & 0 & 0 & 0 & \\
0 & 0 & 0 & 0 & 0 & 0 & 0 & 0 & 0 & 0 & 0 & \\
0 & 0 & 0 & 0 & \mathbf{1} & 0 & 0 & 0 & 0 & 0 & 0 & \\
0 & 0 & 0 & 0 & 0 & 0 & 0 & 0 & 0 & 0 & \mathbf{1} & \\
0 & 0 & 0 & \mathbf{1} & 0 & 0 & 0 & 0 & 0 & 0 & 0 & \\
0 & 0 & 0 & 0 & 0 & 0 & 0 & \mathbf{1} & 0 & 0 & 0 & \\
0 & 0 & 0 & 0 & 0 & \mathbf{1} & 0 & 0 & 0 & 0 & 0 & \\
0 & \mathbf{1} & 0 & 0 & 0 & 0 & 0 & 0 & 0 & 0 & 0 & \\
0 & 0 & \mathbf{1} & 0 & 0 & 0 & 0 & 0 & 0 & 0 & 0 & \\
\mathbf{1} & 0 & 0 & 0 & 0 & 0 & 0 & 0 & 0 & 0 & 0 & \ldots \\
\hline
\end{array}
$$


Let's try something...

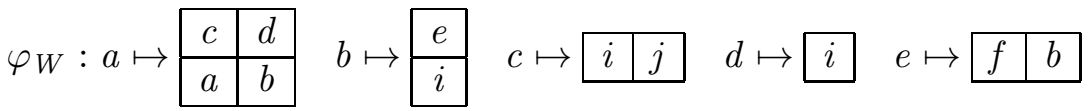

$$
\begin{aligned}
& f \mapsto \begin{array}{|l|l|}
\hline h & d \\
\hline g & b \\
\hline
\end{array} \quad g \mapsto \begin{array}{|l|l|}
\hline h & d \\
\hline f & b \\
\hline
\end{array} \quad h \mapsto \begin{array}{|l|l|}
\hline i & m \\
\hline
\end{array} \quad i \mapsto \begin{array}{|l|l|}
\hline h & d \\
\hline i & m \\
\hline
\end{array}
\end{aligned}
$$

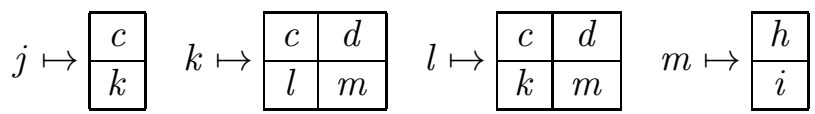

and the coding

$$
\mu_{W}: a, e, g, j, l \mapsto 1, \quad b, c, d, f, h, i, k, m \mapsto 0
$$


The idea:

\section{ShaPe-Symmetric MORPhism (A. MAES, 1999 [47])}

If $P$ is the infinite bidimensional picture that is the fixed point of $\varphi$, then for all $i, j \in \mathbb{N}$, if $\varphi\left(P_{i, j}\right)$ is a block of size $k \times \ell$ then $\varphi\left(P_{j, i}\right)$ is of size $\ell \times k$

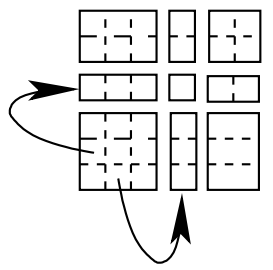


The details: Let $d \geq 2$

A $d$-dimensional picture over $A$ is a map

$$
x: \llbracket 0, s_{1}-1 \rrbracket \times \cdots \times \llbracket 0, s_{d}-1 \rrbracket \rightarrow A
$$

$\left(s_{1}, \ldots, s_{d}\right)$ is the shape of $x$; if $s_{i}<\infty$, for all $i, x$ is bounded. The set of bounded pictures over $A$ is denoted by $\mathcal{B}_{d}(A)$.

If for some $i \in \llbracket 1, d \rrbracket,|x|_{\hat{i}}=|y|_{\hat{i}}=\left(s_{1}, \ldots, s_{i-1}, s_{i+1}, \ldots, s_{d}\right)$, then we define the concatenation of $x$ and $y$ in the direction $i$ to be the $d$-dimensional picture $x \odot^{i} y$ of shape

$$
\left(s_{1}, \ldots, s_{i-1},|x|_{i}+|y|_{i}, s_{i+1}, \ldots, s_{d}\right) .
$$




$$
x=\begin{array}{|l|l|}
\hline a & b \\
\hline c & d \\
\hline
\end{array} \quad \text { and } \quad y=\begin{array}{|l|l|l|}
\hline a & a & b \\
\hline b & c & d \\
\hline
\end{array}
$$

of shape respectively $|x|=(2,2)$ and $|y|=(2,3)$. Since $|x|_{\widehat{2}}=|y|_{\widehat{2}}=2$, we get

$$
x \odot^{2} y=\begin{array}{|l|l|l|l|l|}
\hline a & b & a & a & b \\
\hline c & d & b & c & d \\
\hline
\end{array} .
$$

However $x \odot^{1} y$ is not defined because $2=|x|_{\widehat{1}} \neq|y|_{\widehat{1}}=3$. 
A map $\gamma: A \rightarrow \mathcal{B}_{d}(A)$ cannot necessarily be extended to a morphism $\gamma: \mathcal{B}_{d}(A) \rightarrow \mathcal{B}_{d}(A)$.

$$
\gamma: a \mapsto \begin{array}{|l|l|}
\hline b & d \\
\hline a & a \\
\hline
\end{array}, \quad b \mapsto \begin{array}{|l|}
\hline b \\
\hline c
\end{array}, \quad c \mapsto \begin{array}{l|l|}
a & a
\end{array}, \quad d \mapsto b .
$$

$\odot^{2}:|\gamma(c)|_{\widehat{2}}=|\gamma(d)|_{\widehat{2}}=2,|\gamma(a)|_{\widehat{2}}=|\gamma(b)|_{\widehat{2}}=1$,

$\odot^{1}:|\gamma(c)|_{\widehat{1}}=|\gamma(a)|_{\widehat{1}}=2,|\gamma(d)|_{\widehat{1}}=|\gamma(b)|_{\widehat{1}}=1$.

$$
x=\begin{array}{|l|l|}
\hline c & d \\
\hline a & b \\
\hline
\end{array}, \quad \gamma(x)=\begin{array}{|l|l|l|}
\hline a & a & d \\
\hline b & d & b \\
\hline a & a & c \\
\hline
\end{array}
$$

but $\gamma^{2}(x)$ is not well-defined!

\section{DEFINITION}

If for all $a \in A$ and all $n \geq 1, \gamma^{n}(a)$ is well-defined from $\gamma^{n-1}(a)$, then $\gamma$ is said to be a $d$-dimensional morphism.

$\rightsquigarrow$ the images of any two symbols on a row (resp. column) have the same number of rows (resp. column). 


\section{DEFINITION}

Let $\gamma: \mathcal{B}_{d}(A) \rightarrow \mathcal{B}_{d}(A)$ be a $d$-dimensional morphism having the $d$-dimensional infinite word $x$ as a fixed point.

This word is shape-symmetric with respect to $\gamma$ if, for all permutations $\nu$ of $\llbracket 1, d \rrbracket$, we have, for all $n_{1}, \ldots, n_{d} \geq 0$,

$$
\begin{aligned}
\left|\gamma\left(x\left(n_{1}, \ldots, n_{d}\right)\right)\right| & =\left(s_{1}, \ldots, s_{d}\right) \\
\Downarrow & \\
\left|\gamma\left(x\left(n_{\nu(1)}, \ldots, n_{\nu(d)}\right)\right)\right| & =\left(s_{\nu(1)}, \ldots, s_{\nu(d)}\right) .
\end{aligned}
$$


Reconsider our map $\varphi$ (one can indeed prove that it is a $d$-dimensional morphism having a shape-symmetric fixed point).

$a \mapsto$\begin{tabular}{|c|c|}
\hline$c$ & $d$ \\
\hline $\mathbf{a}$ & $b$ \\
\hline
\end{tabular}$\mapsto$\begin{tabular}{|c|c|c|}
\hline$i$ & $\mathbf{j}$ & $i$ \\
\hline$c$ & $d$ & $\mathbf{e}$ \\
\hline $\mathbf{a}$ & $b$ & $i$ \\
\hline
\end{tabular}$\mapsto$\begin{tabular}{|c|c|c|c|c|}
\hline$h$ & $d$ & $c$ & $h$ & $d$ \\
\hline$i$ & $m$ & $k$ & $i$ & $m$ \\
\hline$i$ & $\mathbf{j}$ & $i$ & $f$ & $b$ \\
\hline$c$ & $d$ & $\mathbf{e}$ & $h$ & $d$ \\
\hline $\mathbf{a}$ & $b$ & $i$ & $i$ & $m$ \\
\hline
\end{tabular}

sizes : $1,2,3,5$ 


$\cdots \mapsto$\begin{tabular}{|c|c|c|c|c|c|c|c|}
\hline$i$ & $m$ & $i$ & $i$ & $\mathbf{j}$ & $i$ & $m$ & $i$ \\
\hline$h$ & $d$ & $h$ & $c$ & $d$ & $h$ & $d$ & $h$ \\
\hline$i$ & $m$ & $i$ & $\mathbf{l}$ & $m$ & $i$ & $m$ & $i$ \\
\hline$h$ & $d$ & $c$ & $h$ & $d$ & $h$ & $d$ & $\mathbf{e}$ \\
\hline$i$ & $m$ & $k$ & $i$ & $m$ & $\mathbf{g}$ & $b$ & $i$ \\
\hline$i$ & $\mathbf{j}$ & $i$ & $f$ & $b$ & $i$ & $m$ & $i$ \\
\hline$c$ & $d$ & $\mathbf{e}$ & $h$ & $d$ & $h$ & $d$ & $h$ \\
\hline $\mathbf{a}$ & $b$ & $i$ & $i$ & $m$ & $i$ & $m$ & $i$ \\
\hline
\end{tabular}

size $: 8, \ldots$ 


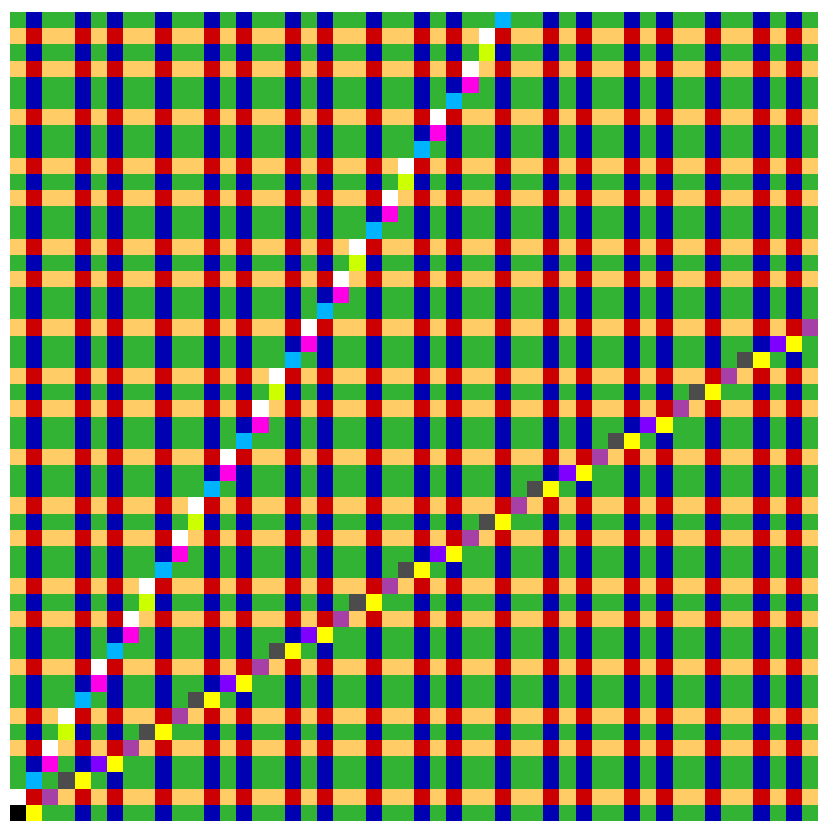

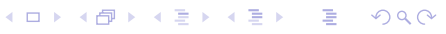


Before proceeding to the proof (of morphic structure of the $\mathcal{P}$-positions of Wythoff)... How did we get that?

\begin{tabular}{|ll|l|ll|ll|ll|ll|l|l}
$\vdots$ & & & & & & & & & & $\vdots$ \\
\hline 0 & 0 & 0 & 0 & 0 & 0 & $\mathbf{1}$ & 0 & 0 & 0 & 0 & \\
\hline 0 & 0 & 0 & 0 & 0 & 0 & 0 & 0 & 0 & 0 & 0 & \\
0 & 0 & 0 & 0 & 0 & 0 & 0 & 0 & 0 & 0 & 0 & \\
\hline 0 & 0 & 0 & 0 & $\mathbf{1}$ & 0 & 0 & 0 & 0 & 0 & 0 & \\
\hline 0 & 0 & 0 & 0 & 0 & 0 & 0 & 0 & 0 & 0 & $\mathbf{1}$ & \\
0 & 0 & 0 & $\mathbf{1}$ & 0 & 0 & 0 & 0 & 0 & 0 & 0 & \\
\hline 0 & 0 & 0 & 0 & 0 & 0 & 0 & $\mathbf{1}$ & 0 & 0 & 0 & \\
0 & 0 & 0 & 0 & 0 & $\mathbf{1}$ & 0 & 0 & 0 & 0 & 0 & \\
\hline 0 & $\mathbf{1}$ & 0 & 0 & 0 & 0 & 0 & 0 & 0 & 0 & 0 & \\
\hline 0 & 0 & $\mathbf{1}$ & 0 & 0 & 0 & 0 & 0 & 0 & 0 & 0 & \\
$\mathbf{1}$ & 0 & 0 & 0 & 0 & 0 & 0 & 0 & 0 & 0 & 0 & $\ldots$ \\
\hline
\end{tabular}

shape sequence: $2122121221 \ldots$ 


\section{THEOREM 觜[43]}

The morphism $\varphi_{W}$ and the coding $\mu_{W}$ give the 2-dimensional infinite word coding the $\mathcal{P}$-positions of Wythoff.

Proof. We can do the same ${ }^{4}$ as for the uni-dimensional case: We associate with $\varphi$ an automaton with input alphabet

$$
\begin{gathered}
\left\{\left(\begin{array}{l}
0 \\
0
\end{array}\right),\left(\begin{array}{l}
1 \\
0
\end{array}\right),\left(\begin{array}{l}
0 \\
1
\end{array}\right),\left(\begin{array}{l}
1 \\
1
\end{array}\right)\right\} \\
\varphi(r)=\begin{array}{|l|l|}
u & v \\
\hline s & t \\
\hline
\end{array}, \begin{array}{|l|l|}
\hline s & t \\
\hline s
\end{array} \quad \text { or } \begin{array}{|l|}
s \\
\hline
\end{array}
\end{gathered}
$$

we have transitions like

$$
r \stackrel{\left(\begin{array}{l}
0 \\
0
\end{array}\right)}{\longrightarrow} s, \quad r \stackrel{\left(\begin{array}{l}
1 \\
0
\end{array}\right)}{\longrightarrow} t, \quad r \stackrel{\left(\begin{array}{l}
0 \\
1
\end{array}\right)}{\longrightarrow} u, \quad r \stackrel{\left(\begin{array}{l}
1 \\
1
\end{array}\right)}{\longrightarrow} v .
$$

${ }^{4}$ Similar to the general theorem: the $n$th symbol is obtained by feeding a DFA with the $n$th word of the accepted language. 
From morphism to automaton, we get

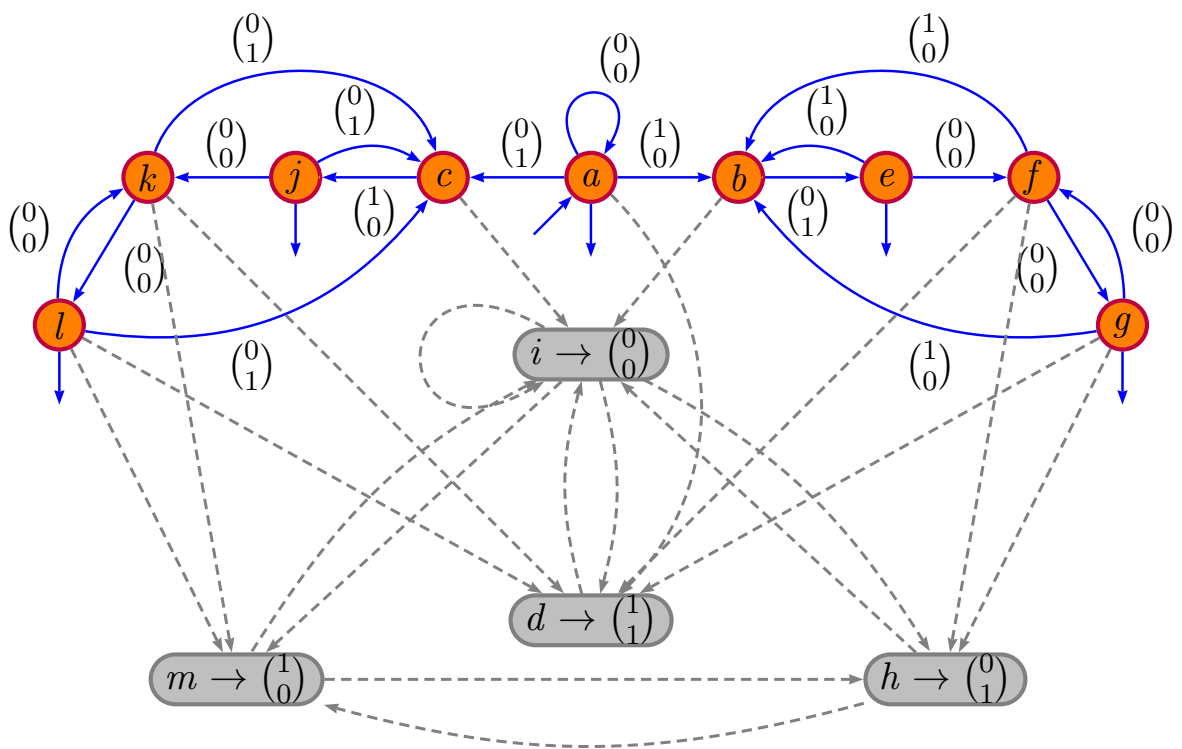


1) If all states are assumed to be final, this automaton accepts the words

$$
\left(\begin{array}{l}
u \\
v
\end{array}\right)
$$

where $|u|=|v|$ and $u, v$ are both valid $F$-representation (possibly padded with zeroes).

2) If we restrict to the "blue" part, this automaton accepts the words

$$
\left(\begin{array}{l}
0 w_{1} \cdots w_{\ell} \\
w_{1} \cdots w_{\ell} 0
\end{array}\right) \text { and }\left(\begin{array}{c}
w_{1} \cdots w_{\ell} 0 \\
0 w_{1} \cdots w_{\ell}
\end{array}\right)
$$

where $w_{1} \cdots w_{\ell}$ is a valid $F$-representation.

3) Now, if the set of final states is $\{a, e, g, j, l\}$, we have the extra condition that $w_{1} \cdots w_{\ell}$ ends with an even number of zeroes.

With our previous characterization of $\mathcal{P}$-positions, this concludes the proof. 

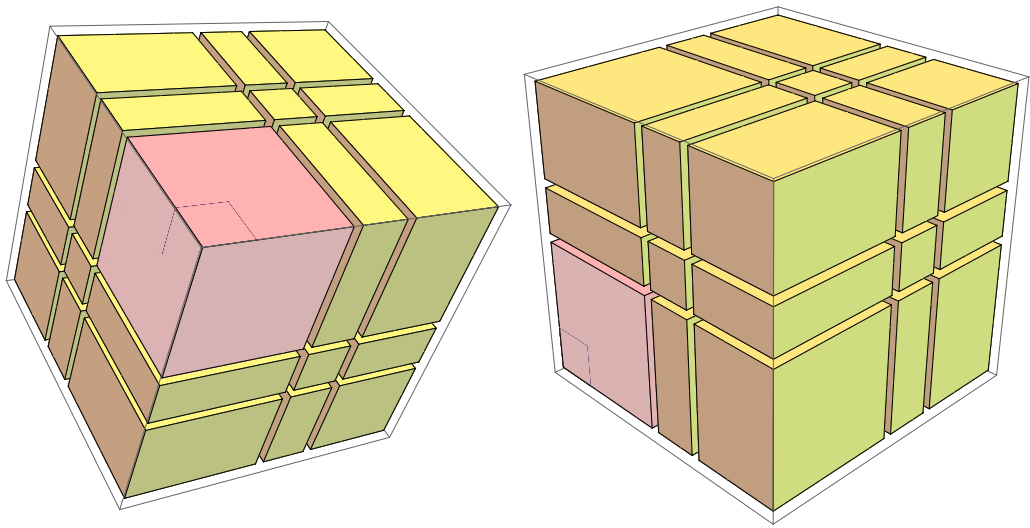

Initial blocks of some 3-dimensional shape-symmetric picture [47, Maes' thesis p. 107]. 


\section{ASSOCIATED DECISION PROBLEMS}

Faculté des Sciences

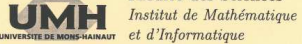

Service de Logique Mathématique et Algèbre

MORPHIC PREDICATES

AND APPLICATIONS

TO THE DECIDABILITY

OF ARITHMETIC THEORIES

Arnaud MaEs

Dissertation originale présentés

pour Fobtention du grade académique de

Docteur EN SCIENCES

Janvier 1999
Some Results from Maes' papers:

- Determining whether or not a map $\mu: \mathcal{B}_{d}(A) \rightarrow \mathcal{B}_{d}(A)$ is a $d$-dimensional morphism is a decidable problem.

- If $\mu$ is prolongable on a letter $a$, then it is decidable whether or not the fixed point $\mu^{\omega}(a)$ is shape-symmetric.

$[45,46,47]$ 


\section{ADDING OR REMOVING MOVES}

$\rightsquigarrow$ How can we alter the set of moves 紫 to keep the same set of $\mathcal{P}$-positions?

\section{REMARK}

This means that several rule-sets (different "games") could lead to the same set of $\mathcal{P}$-positions.

In a subtraction game, observe that a move can be adjoined (without altering the set of $\mathcal{P}$-positions) if and only if it does not belong to $\mathcal{P}-\mathcal{P}$.

\section{Corollary}

We can adjoined the move $(i, j)_{i<j}$ to Wythoff's rule-set iff

$$
\begin{gathered}
(i, j) \neq\left(\lfloor n \varphi\rfloor-\lfloor m \varphi\rfloor,\left\lfloor n \varphi^{2}\right\rfloor-\left\lfloor m \varphi^{2}\right\rfloor\right) \forall n>m \geq 0 \\
\text { and }(i, j) \neq\left(\lfloor n \varphi\rfloor-\left\lfloor m \varphi^{2}\right\rfloor,\left\lfloor n \varphi^{2}\right\rfloor-\lfloor m \varphi\rfloor\right) \forall n>m \geq 0 .
\end{gathered}
$$




\section{TheOREM (E. Duchêne ET AL. 2010 [43]}

$(i, j)_{i<j}$ may be adjoined iff there exist valid $F$-representations $u, u^{\prime}$ such that one the three properties is satisfied :

- $\left(\operatorname{rep}_{F}(i-1), \operatorname{rep}_{F}(j-1)\right)=(u 0, u 01)$

- $\left(\operatorname{rep}_{F}(i-2), \operatorname{rep}_{F}(j-2)\right)=(u 0, u 01)$

- $\left(\operatorname{rep}_{F}(j-\lfloor i \varphi\rfloor-2), \operatorname{rep}_{F}(j-\lfloor i \varphi\rfloor-2+i)\right)=\left(u 1, u^{\prime} 0\right)$;

\begin{tabular}{|llllllllllll}
0 & 0 & 0 & 1 & 0 & 0 & 1 & 0 & 0 & 0 & 0 & \\
0 & 1 & 0 & 0 & 1 & 0 & 0 & 0 & 0 & 0 & 0 & \\
0 & 0 & 0 & 0 & 0 & 1 & 0 & 0 & 0 & 0 & 0 & \\
0 & 1 & 0 & 0 & 1 & 0 & 0 & 0 & 0 & 0 & 0 & \\
0 & 0 & 1 & 0 & 1 & 0 & 0 & 0 & 0 & 0 & 1 & \\
0 & 0 & 0 & 1 & 0 & 0 & 0 & 0 & 1 & 0 & 0 & \\
0 & 1 & 0 & 0 & 0 & 0 & 1 & 1 & 0 & 1 & 0 & \\
0 & 0 & 1 & 0 & 0 & 1 & 0 & 0 & 0 & 0 & 1 & \\
0 & 1 & 0 & 1 & 0 & 0 & 1 & 0 & 0 & 0 & 0 & \\
0 & 0 & 1 & 0 & 1 & 0 & 0 & 1 & 0 & 1 & 0 & \\
0 & 0 & 0 & 0 & 0 & 0 & 0 & 0 & 0 & 0 & 0 & $\cdots$ \\
\hline
\end{tabular}


- There is no redundant move in Wythoff's game.

Question: Does the infinite 2-dimensional word on the previous slide have a shape-symmetric morphic structure?

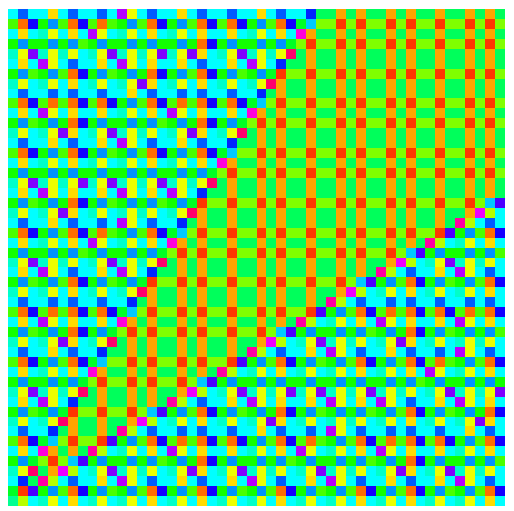

We conjectured a morphism over 26 letters. 
Another direction leads to the concept of invariant games [48, 49].

A game $G: \mathbb{N}^{n} \rightarrow 2^{\mathbb{N}^{n}}$ (assigning each position to a set of available moves) is invariant if there exists a set $I \subseteq \mathbb{N}^{n}$ such that, for all positions $\mathbf{p}$, we have

$$
G(\mathbf{p})=I \cap\left\{\mathbf{m} \in \mathbb{N}^{n} \mid \mathbf{m} \leq \mathbf{p}\right\} .
$$

Otherwise stated, we may apply exactly the same moves to every position, with the only restriction that there are enough tokens left.

\section{EXAMPLE}

The game of Nim is invariant:

$$
I_{\mathrm{NIM}}=\{(i, 0) \mid i \geq 1\} \cup\{(0, j) \mid j \geq 1\} .
$$

Wythoff's game is invariant:

$$
I_{\mathrm{WYTHOFF}}=I_{\mathrm{NIM}} \cup\{(k, k) \mid k \geq 1\} .
$$


For an example of non-invariant game, consider the following map,

$$
\begin{gathered}
G_{\mathrm{EVEN}}: \mathbb{N}^{2} \rightarrow 2^{\mathbb{N}^{2},} \\
(x, y) \mapsto \begin{cases}\{(i, 0) \mid i \in \llbracket 1, x \rrbracket\}, & \text { if } x+y \text { is even; } \\
\{(i, i) \mid i \in \llbracket 1, \min \{x, y\} \rrbracket\}, & \text { otherwise. }\end{cases}
\end{gathered}
$$




\section{LINK WITH ANS}

Actually, we have already seen Abstract Numeration Systems...

\section{DEFINITION $[50]$}

An abstract numeration system $\mathcal{S}=(L, A,<)$ is a regular language $L$ over a totally ordered finite alphabet $(A,<)$.

- Enumerating the words in $L$ using genealogical ordering provides a one-to-one correspondence between $\mathbb{N}$ and $L$ :

$$
\operatorname{rep}_{\mathcal{S}}: \mathbb{N} \rightarrow L, \quad \operatorname{val}_{\mathcal{S}}: L \rightarrow \mathbb{N} .
$$

- This generalizes any positional system $U$ for which $\operatorname{rep}_{U}(\mathbb{N})$ is regular.

P. Lecomte, M.R., Numeration systems on a regular language, Theory

Comput. Syst. 34 (2001), 27-44. 


\section{ABSTRACT NUMERATION SYSTEMS}

Example : consider a prefix-closed language $L=\{b, \varepsilon\}\{a, a b\}^{*}$

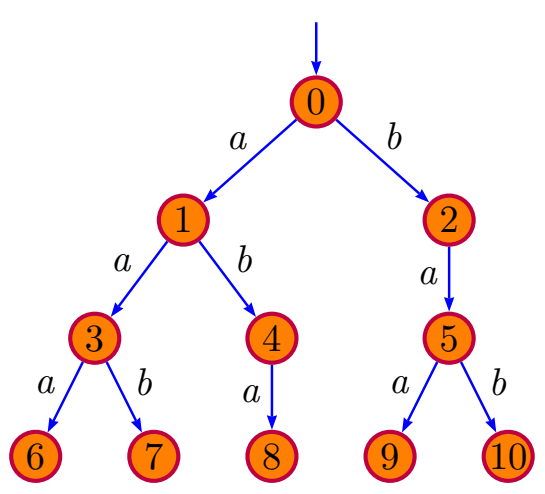

\begin{tabular}{r|r}
$n$ & $\operatorname{rep}_{\mathcal{S}}(n)$ \\
\hline 0 & $\varepsilon$ \\
1 & $a$ \\
2 & $b$ \\
3 & $a a$ \\
4 & $a b$ \\
5 & $b a$ \\
6 & $a a a$ \\
7 & $a a b$ \\
8 & $a b a$ \\
9 & $b a a$ \\
10 & $b a b$
\end{tabular}




\section{ABSTRACT NUMERATION SYSTEMS}

A non-positional ANS $L=a^{*} b^{*}$ \#b

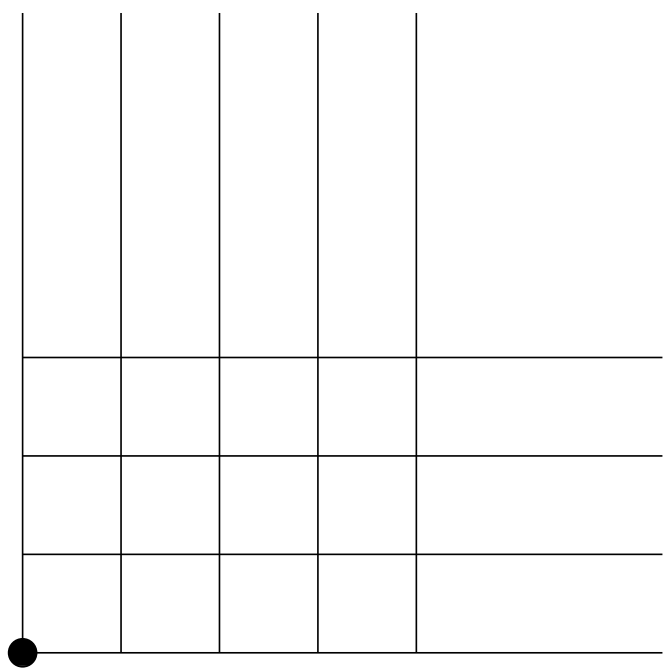

\#a 


\section{ABSTRACT NUMERATION SYSTEMS}

A non-positional ANS $L=a^{*} b^{*}$ \#b

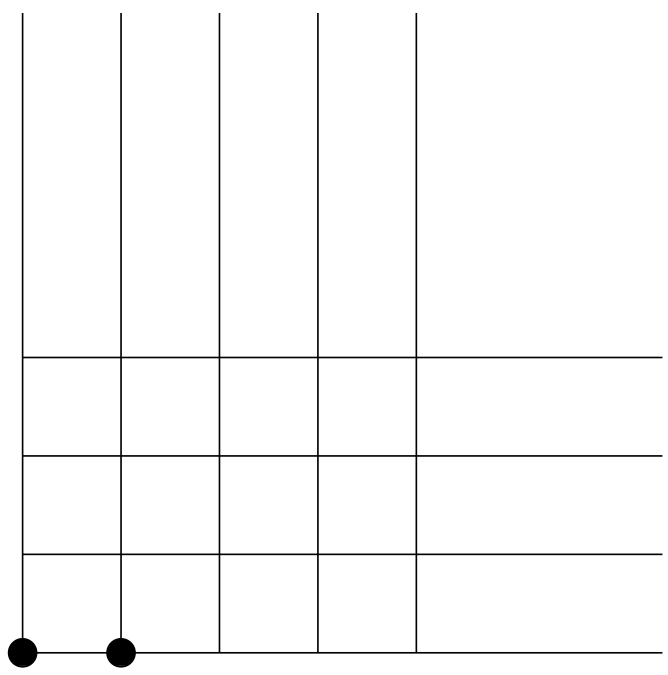

\#a 


\section{ABSTRACT NUMERATION SYSTEMS}

A non-positional ANS $L=a^{*} b^{*}$ \#b

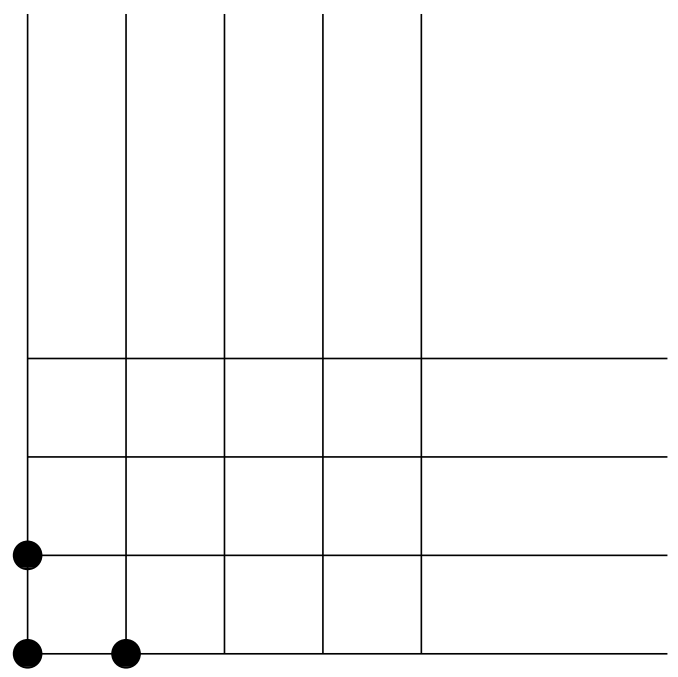

\#a 


\section{ABSTRACT NUMERATION SYSTEMS}

A non-positional ANS $L=a^{*} b^{*}$ \#b

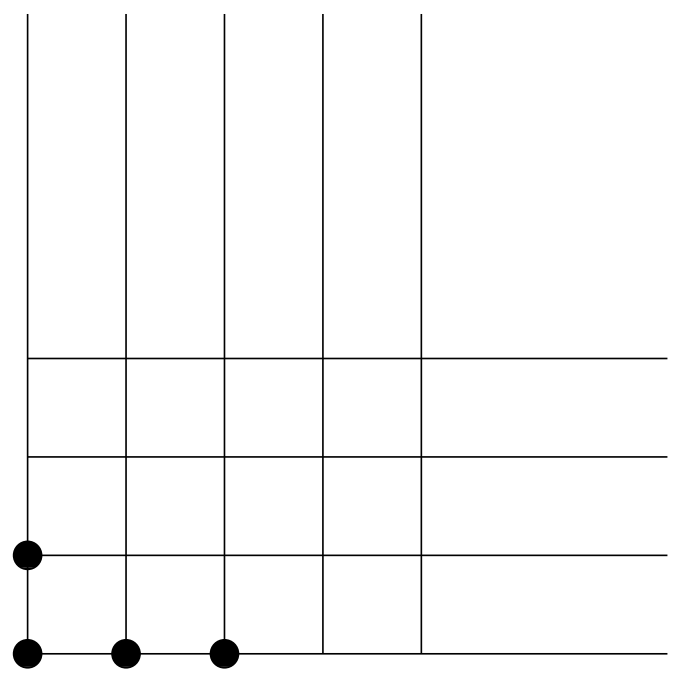

\#a 


\section{ABSTRACT NUMERATION SYSTEMS}

A non-positional ANS $L=a^{*} b^{*}$ \#b

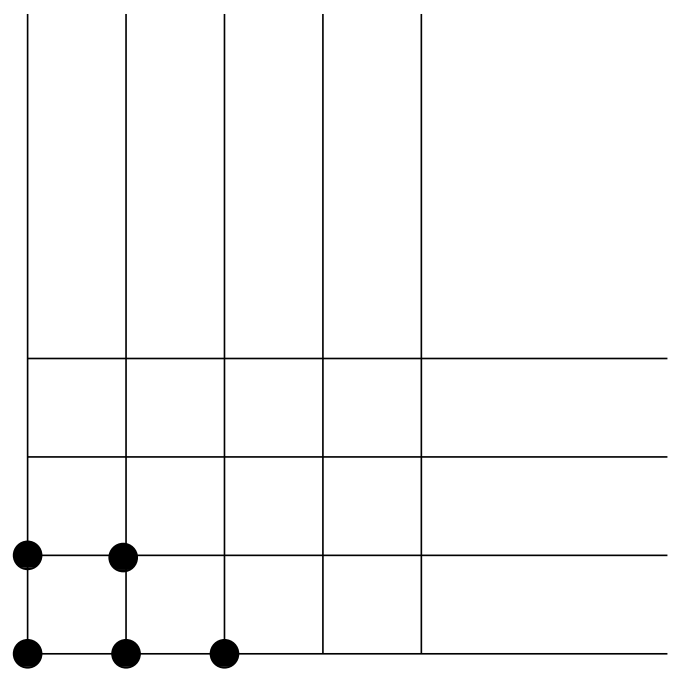

\#a 


\section{ABSTRACT NUMERATION SYSTEMS}

A non-positional ANS $L=a^{*} b^{*}$ \#b

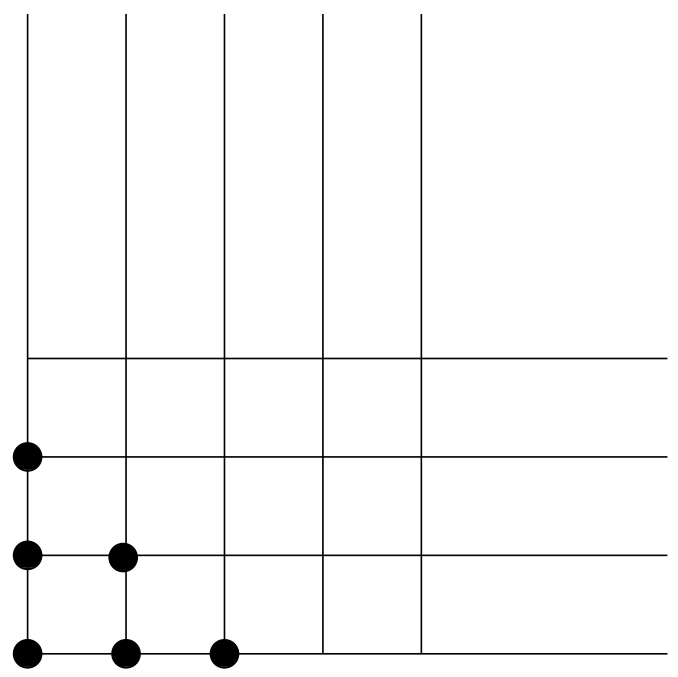

\#a 


\section{ABSTRACT NUMERATION SYSTEMS}

A non-positional ANS $L=a^{*} b^{*}$ \#b

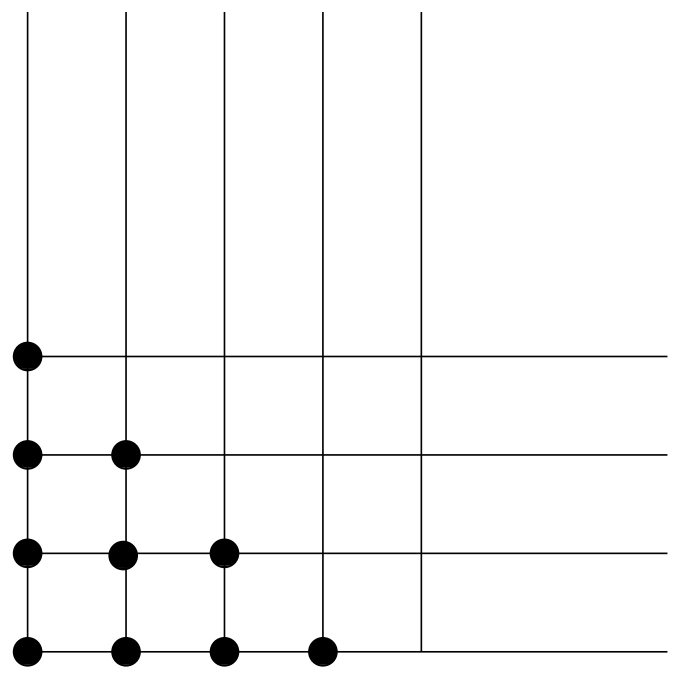

\#a 


\section{ABSTRACT NUMERATION SYSTEMS}

A non-positional ANS $L=a^{*} b^{*}$ \#b

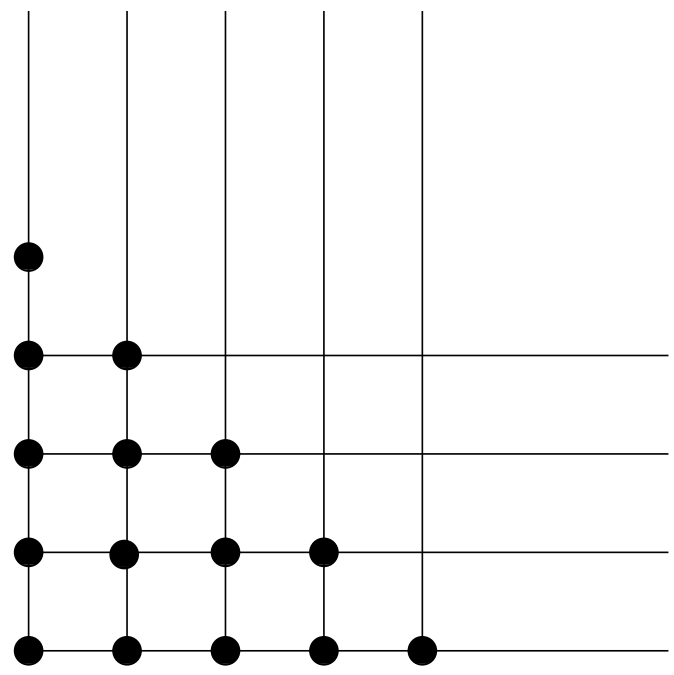

\begin{tabular}{r|r}
$n$ & $\operatorname{rep}_{\mathcal{S}}(n)$ \\
\hline 0 & $\varepsilon$ \\
1 & $a$ \\
2 & $b$ \\
3 & $a a$ \\
4 & $a b$ \\
5 & $b b$ \\
6 & $a a a$ \\
7 & $a a b$ \\
8 & $a b b$ \\
9 & $b b b$ \\
10 & $a a a a$
\end{tabular}

\#a 


\section{ABSTRACT NUMERATION SYSTEMS}

A non-positional ANS $L=a^{*} b^{*}$

$$
\begin{aligned}
& \operatorname{val}_{\mathcal{S}}\left(a^{p} b^{q}\right)=\frac{1}{2}(p+q)(p+q+1)+q=\left(\begin{array}{c}
p+q+1 \\
2
\end{array}\right)+\left(\begin{array}{l}
q \\
1
\end{array}\right) \\
& \begin{array}{cccccccc}
\varepsilon & a & b & a a & a b & b b & a a a & \cdots \\
\hline 0 & 1 & 2 & 3 & 4 & 5 & 6 & \cdots
\end{array} \\
& U_{0}=1, \quad U_{1}=2, p(a)=1, p(b)=2
\end{aligned}
$$

$$
\forall n \in \mathbb{N}, \exists z_{1}, \ldots, z_{\ell}: n=\left(\begin{array}{c}
z_{\ell} \\
\ell
\end{array}\right)+\left(\begin{array}{c}
z_{\ell-1} \\
\ell-1
\end{array}\right)+\cdots+\left(\begin{array}{c}
z_{1} \\
1
\end{array}\right)
$$

with the condition $z_{\ell}>z_{\ell-1}>\cdots>z_{1} \geq 0$ 
There was already some form of abstract numeration system in Maes' Ph.D. thesis (1999) [47].

Rem. 6.9, p. 134, "The set of codes of $\mathbb{N}$ given by the above automaton is of course a regular language ... The language read by $A$ is $0^{*} L$. However, the above coding is not a numeration system in the sense of [6]. Indeed, the representation of a natural number is not obtained using a 'Euclidian division' algorithm." 


\section{$\mathcal{S}$-Automatic Sequences}

Two ingredients: an ANS $S=\left(a^{*} b^{*}, a<b\right)$ and a DFAO $\mathcal{M}$ [39]

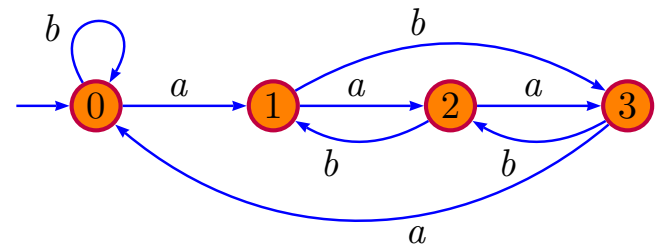

$$
x_{n}=\mathcal{M} \cdot \operatorname{rep}_{S}(n)
$$

$\mathbf{x}=01023031200231010123023031203120231002310123010123 \ldots$ 
$S=\left(a^{*} b^{*}, a<b\right)$ padding symbol: \#

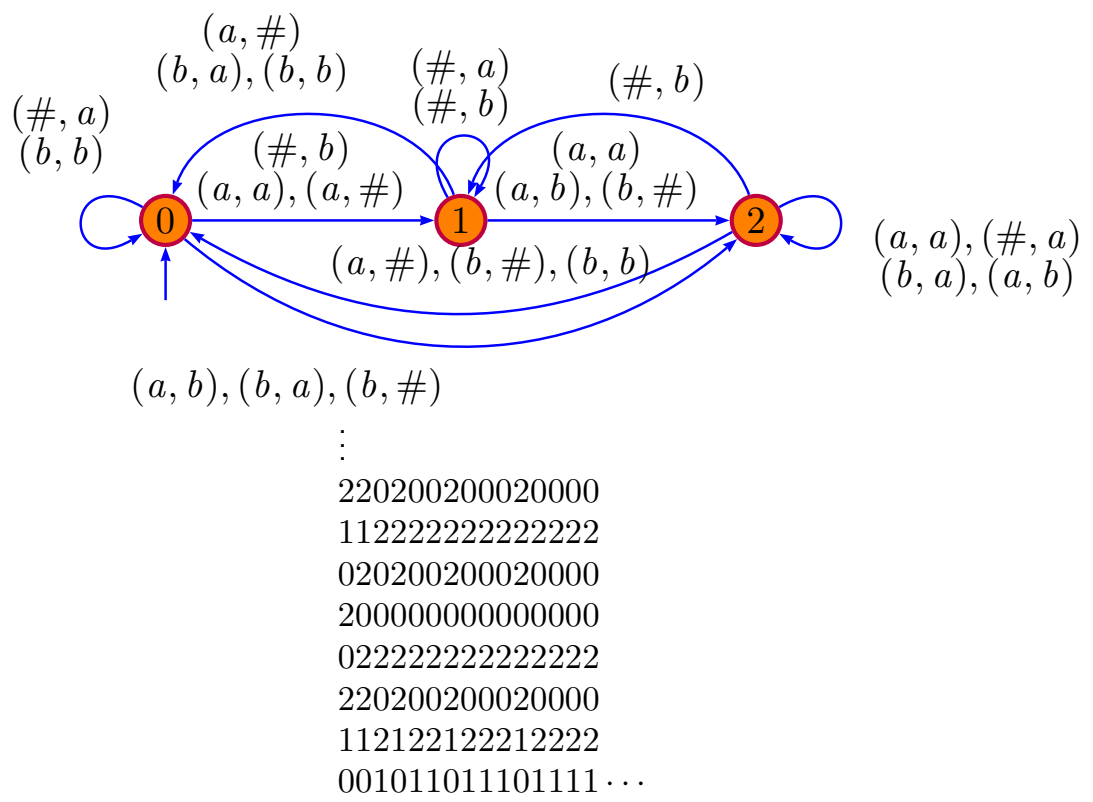




\section{Theorem (A. MAEs, M.R. [40]}

An infinite word is morphic if and only if it is $S$-automatic for some abstract numeration system $S$.

$\Rightarrow$ Already proven.

$\Leftarrow$ We need to get rid off erasing morphisms.

Simulate the product of the two automata.

\section{Theorem (É. Charlier, T. Kärki, M.R. [54])}

Let $d \geq 1$. The $d$-dimensional infinite word $x$ is $S$-automatic, for some abstract numeration system $S=(L, \Sigma,<)$ where $\varepsilon \in L$, if and only if $x$ is the image by a coding of a shape-symmetric infinite $d$-dimensional word. 


\section{SUMMARY}

$k$-automatic sequence<smiles>[AlH2]</smiles>

$k$-uniform morphism

+ coding

[A. Cobham'72] [8]
$\mathcal{S}$-automatic sequence

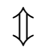

non-erasing morphism

+ coding

[A. Maes, M.R.'02] [39, 40]

multidimensional setup

$$
x: \mathbb{N}^{d} \rightarrow A
$$

$k$-automatic sequence

Æ

morphism $g: A \rightarrow\left(A^{k}\right)^{d}$

+ coding

[O. Salon' 87] [44]

$\mathcal{S}$-automatic sequence

$\mathbb{1}$

"shape-symmetric" morphism

+ coding

[É. Charlier, T. Kärki, M.R.'09] [54] 


\section{GAMES WiTh A FINITE SET OF MOVES}

Games like Nim or Wythoff have an infinite set of moves.

\section{IN ONE DIMENSION [1]}

Every (invariant) finite subtraction game on one pile, i.e., $I \subset \mathbb{N}$ is finite, has an ultimately periodic Grundy function.

Proof:

Let $m=\# I$ (max. number of options), then $\mathcal{G}(n) \leq m$ for all $n$.

Let $k=\max I$, there are $(m+1)^{k}$ possible $k$-tuples taking values in $\{0, \ldots, m\}$. $\mathcal{G}(n)$ depends only on $\mathcal{G}(n-i)$ for $1 \leq i \leq k$.

Hence, there exist $i<j$

$$
\mathcal{G}(i+n)=\mathcal{G}(j+n) \text { for all } n \in\{0, \ldots, k-1\} .
$$

Thus $j-i$ is a period of $\mathcal{G}$ with preperiod $i$. 
Another similar result

\section{[1, A. Siegel, P. 188]}

Consider an (invariant) finite subtraction game on one pile, with $I \subset \mathbb{N}$ as set of moves. If there exist $N \geq 0$ and $p \geq 1$ such that

$$
\mathcal{G}(n+p)=\mathcal{G}(n), \quad \forall N \leq n<N+\max I
$$

then $\mathcal{G}(n+p)=\mathcal{G}(n)$ for all $n \geq N$. 
If we may optionally split a pile...

Definition from Wikipedia:

An octal game is played with tokens divided into heaps.

Two players take turns moving until no moves are possible.

Every move consists of selecting just one of the heaps, and either

- removing all of the tokens in the heap, leaving no heap,

- removing some but not all of the tokens, leaving one smaller heap, or

- removing some of the tokens and dividing the remaining tokens into two nonempty heaps.

Heaps other than the selected heap remain unchanged.

The last player to move wins in normal play. 
Coding of an octal game (Conway code)

$$
d_{0} \bullet d_{1} d_{2} d_{3} \cdots \quad d_{i} \in\{0, \ldots, 7\}
$$

$d_{i}$ written in base 8: $e_{2}^{(i)} e_{1}^{(i)} e_{0}^{(i)}$ gives the conditions under which $i$ token may be removed.

- $e_{0}^{(i)}=1$, then a (full) heap with $i$ token can be suppressed

- $e_{1}^{(i)}=1$, then a heap with $n>i$ token can be replaced with a heap with $n-i$ token left

- $e_{2}^{(i)}=1$, then a heap with $n$ token can be replaced with two heaps containing respectively $a$ and $b$ token, $a, b \geq 1$, $a+b=n-i$.

\section{EXAMPLE}

The game of NIM is coded by $0 \bullet 3333 \cdots, \operatorname{rep}_{8}(3)=011$.

A finite subtraction game $I=\{3,5,6\}$ is of the form $0 \bullet 003033$. 


\section{THEOREM (OCTAL GAME PERIODICITY [1])}

Consider a finite octal game $d_{0} \bullet d_{1} d_{2} \cdots d_{k}$. If there exist $N \geq 0$ and $p \geq 1$ such that

$$
\mathcal{G}(n+p)=\mathcal{G}(n), \quad \forall N \leq n<2 N+p+\max I
$$

then $\mathcal{G}(n+p)=\mathcal{G}(n)$ for all $n \geq N$.

Are all finite octal games ultimately periodic? [55, R. Guy]

$0 \bullet 07$ has period 34 and preperiod 53 [Guy, Smith 1956]

$0 \bullet 007$ no known periodicity...

$0 \bullet 165$ has period 1550 and preperiod 5181 [56, Austin, 1976]

$0 \bullet 106$ has period $\simeq 3.10^{11}$ and preperiod $\simeq 4.10^{11}$

[Flammenkamp, 2002]

http://wwwhomes. uni-bielefeld.de/achim/octal.html 
With more than one pile (let's say two piles).

\section{NATURAL QUESTION}

What is the structure of the $\mathcal{G}$-values for a finite subtraction game over $k$ piles? Do we get a Presburger definable set, i.e., each value determines a semi-linear set?

Cobham-Semenov' theorem: Let $p, q \geq 2$ be multiplicatively independent integers. If $X \subset \mathbb{N}^{k}$ is both $p$-recognizable and $q$-recognizable, then it is definable by a first-order formula in the Presburger arithmetic $\langle\mathbb{N},+\rangle[10]$.

Work in progress: X. Badin De Montjoye, V. Gledel, V. Marsault, A. Massuir, M.R. 
If we let $I=\{(2,1),(3,5)\}$, we get

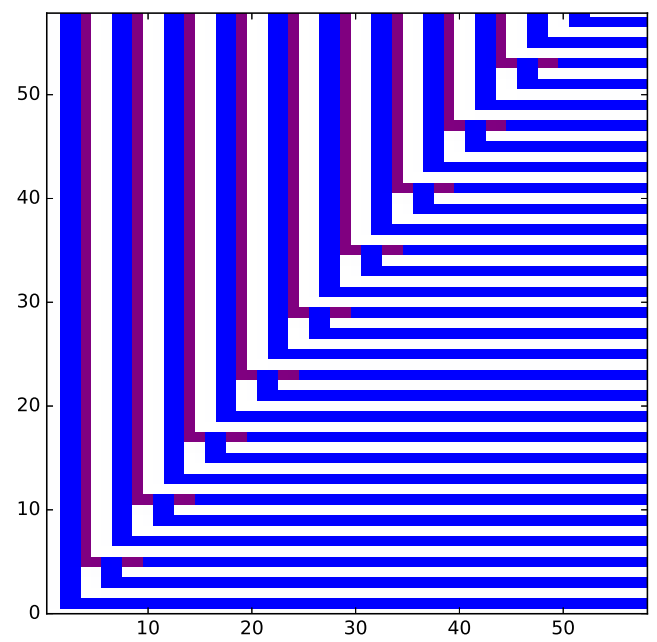

\section{PROPOSITION}

If $\# I=2$, then the set of $\mathcal{G}$-values is Presburger definable. 
If we let $I=\{(1,3),(3,1),(4,4)\}$, we get

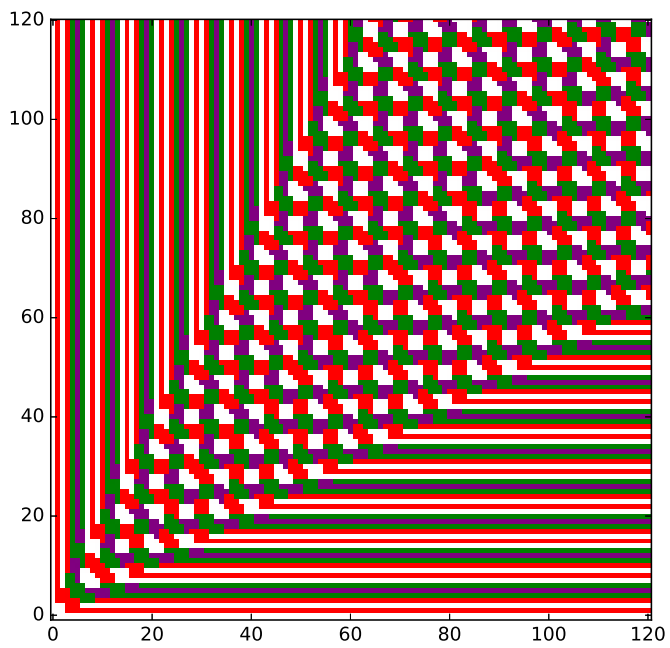


If we let $I=\{(1,2),(2,1),(3,5),(5,3)\}$, we get

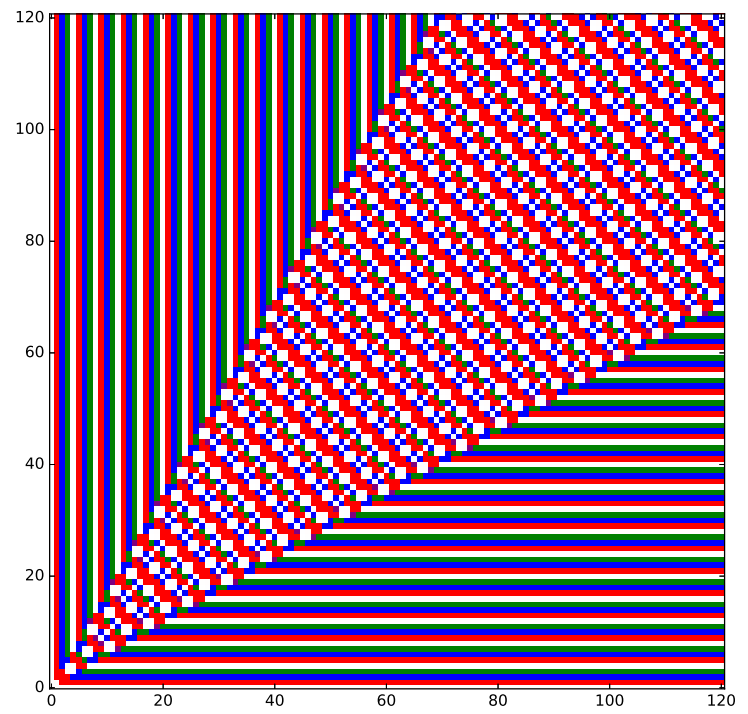


If we let $I=\{(1,2),(2,1),(3,5),(5,3),(2,2)\}$, we get

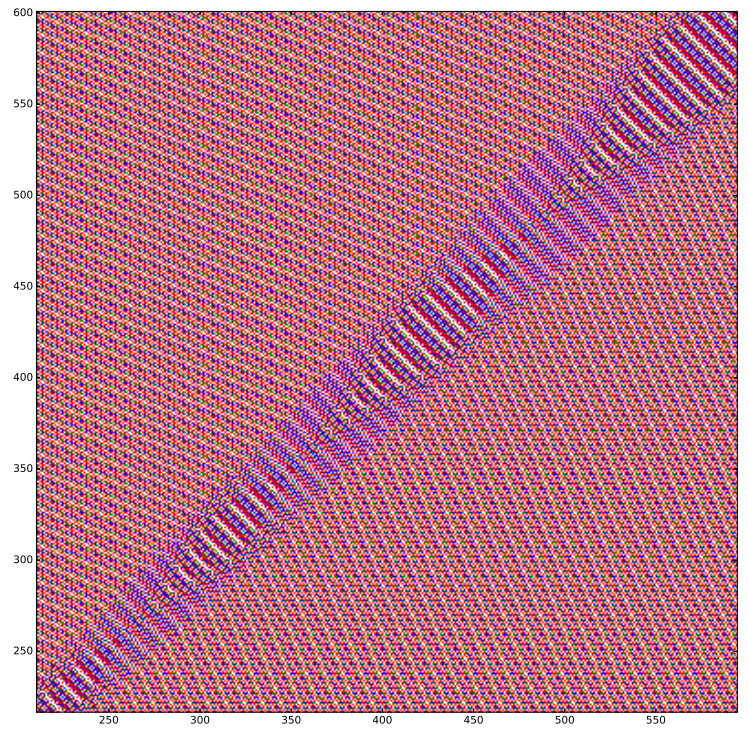

We think that this one is NOT Presburger definable. 
If we let $I=\{(10,2),(2,10),(32,5),(5,32),(10,10)\}$, we get

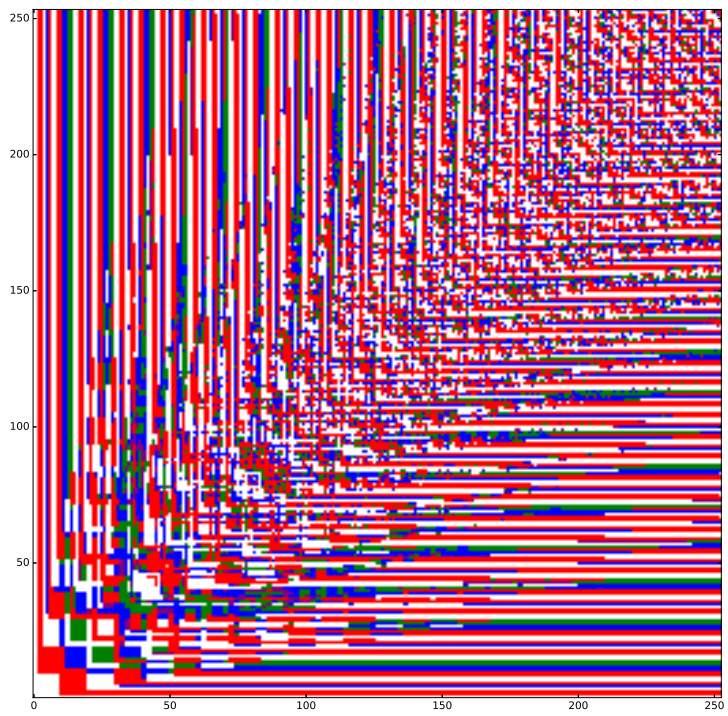


If we let $I=\{(10,2),(2,10),(32,5),(5,32),(10,10)\}$, we get

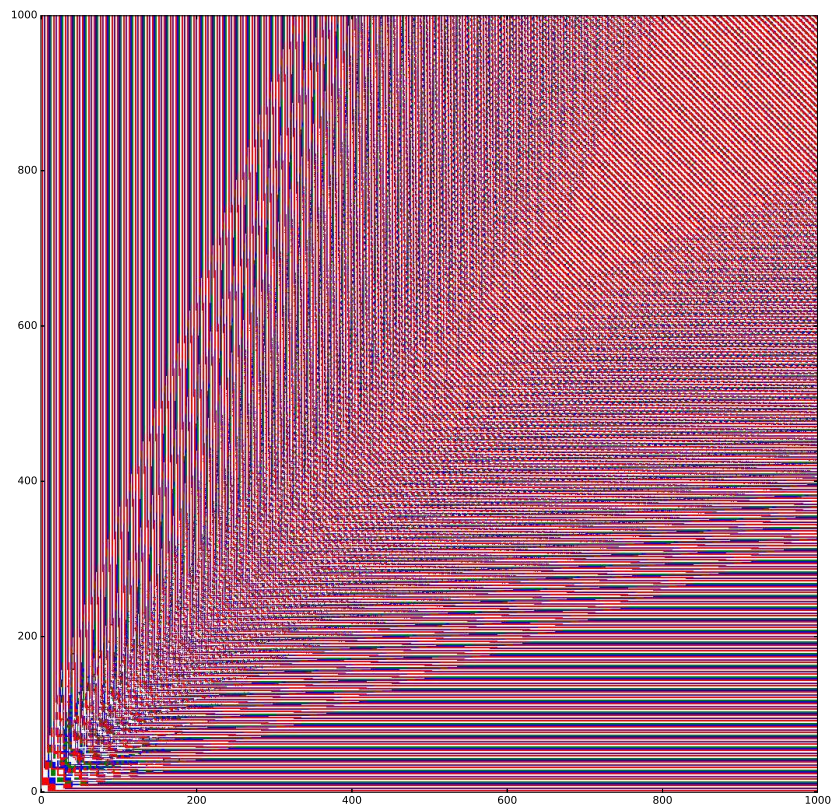


Cellular automata - kind of space-time diagram with bounded memory, the rules are $(1,2)$ and $(3,1)$

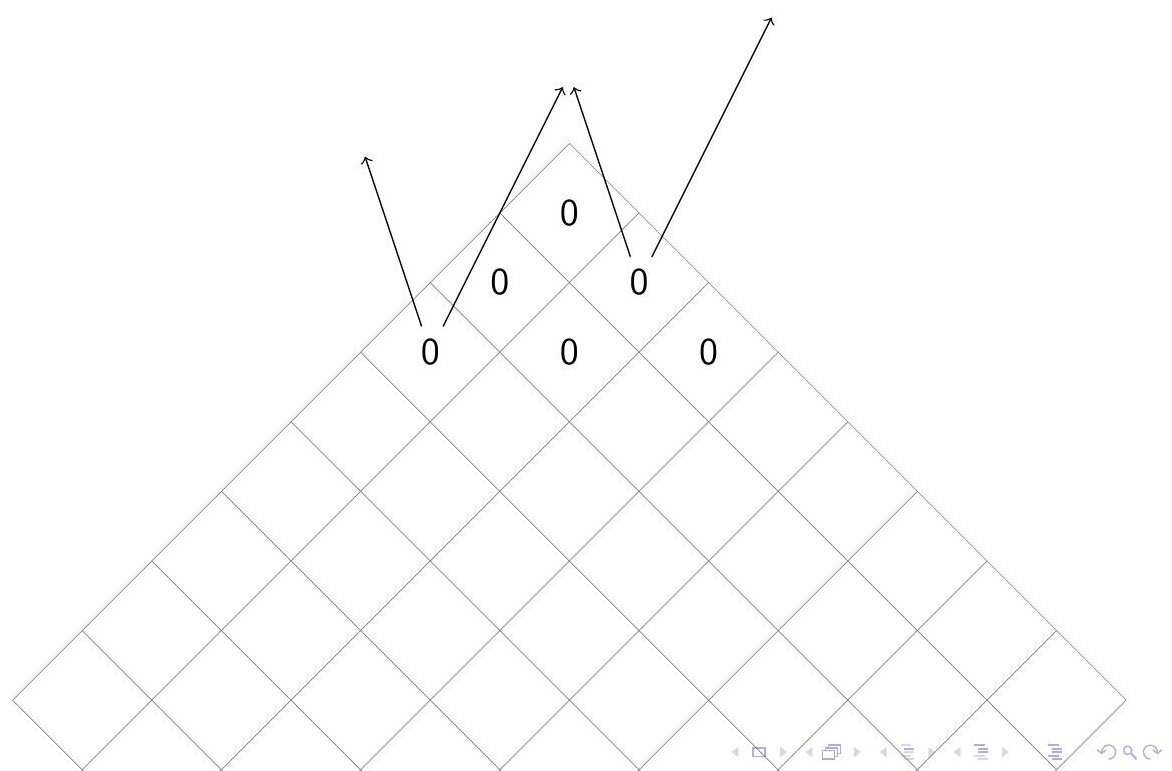


Cellular automata - kind of space-time diagram with bounded memory, the rules are $(1,2)$ and $(3,1)$

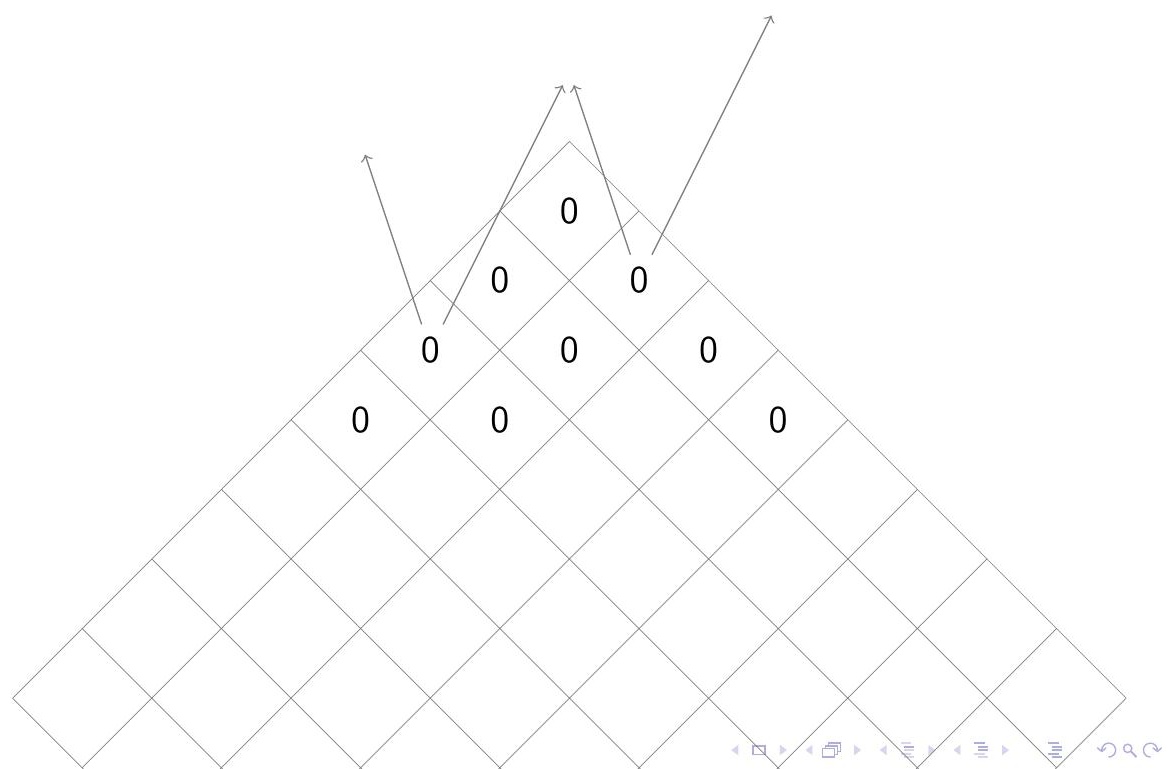


Cellular automata - kind of space-time diagram with bounded memory, the rules are $(1,2)$ and $(3,1)$

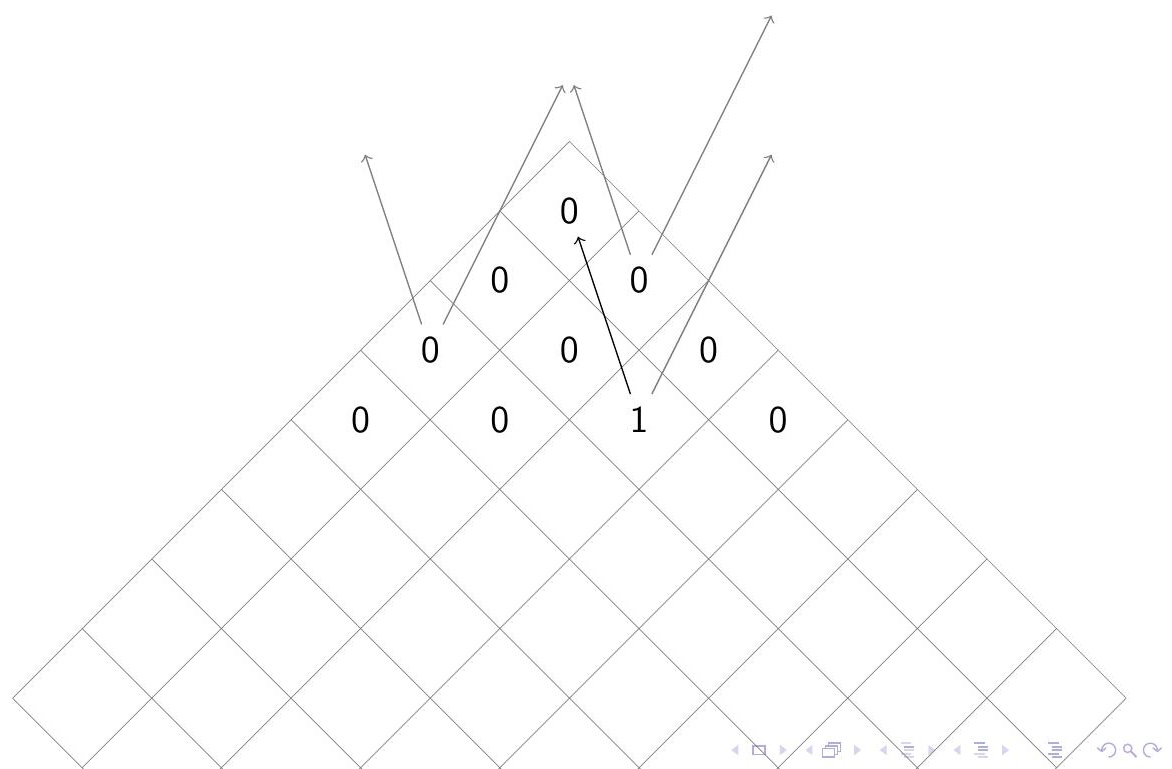


Cellular automata - kind of space-time diagram with bounded memory, the rules are $(1,2)$ and $(3,1)$

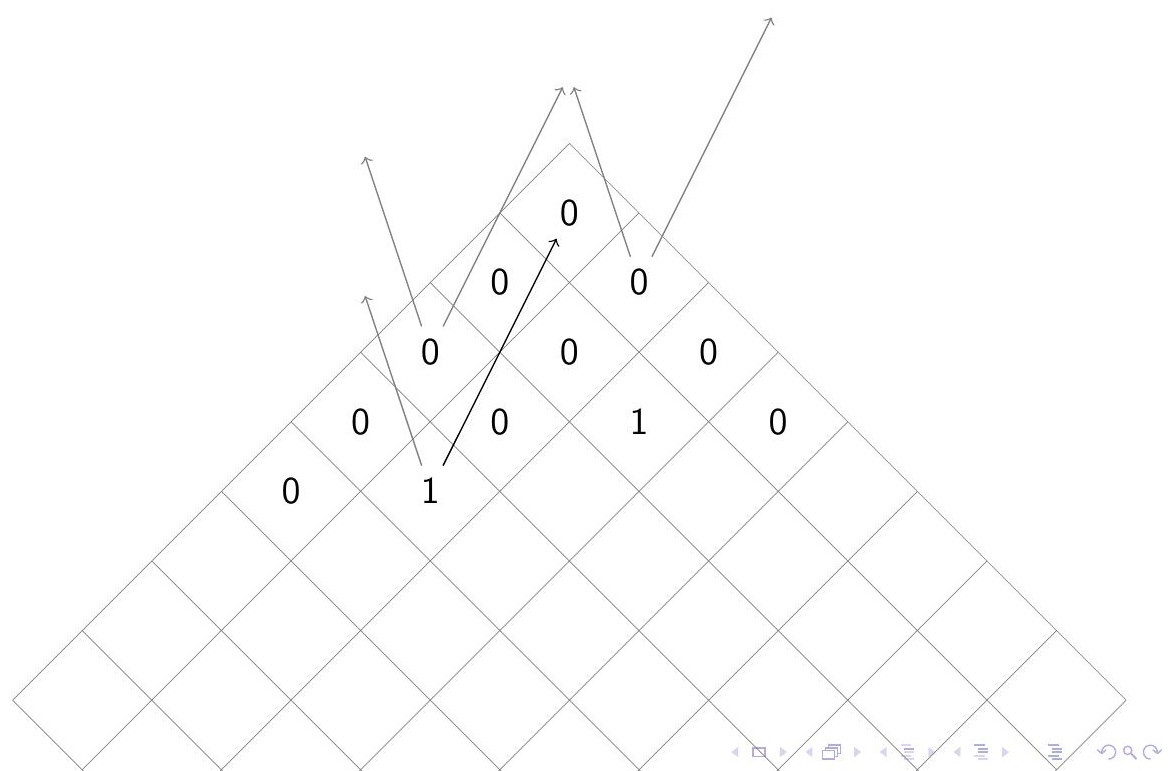


Cellular automata - kind of space-time diagram with bounded memory, the rules are $(1,2)$ and $(3,1)$

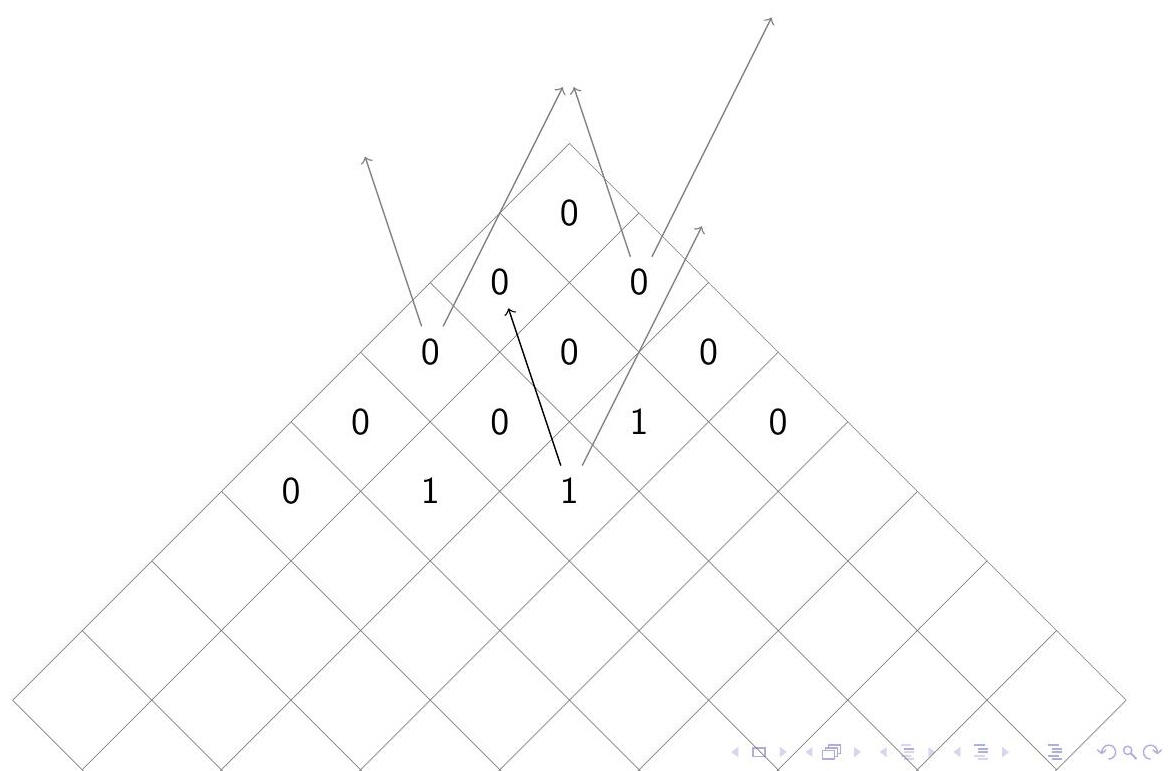


Cellular automata - kind of space-time diagram with bounded memory, the rules are $(1,2)$ and $(3,1)$

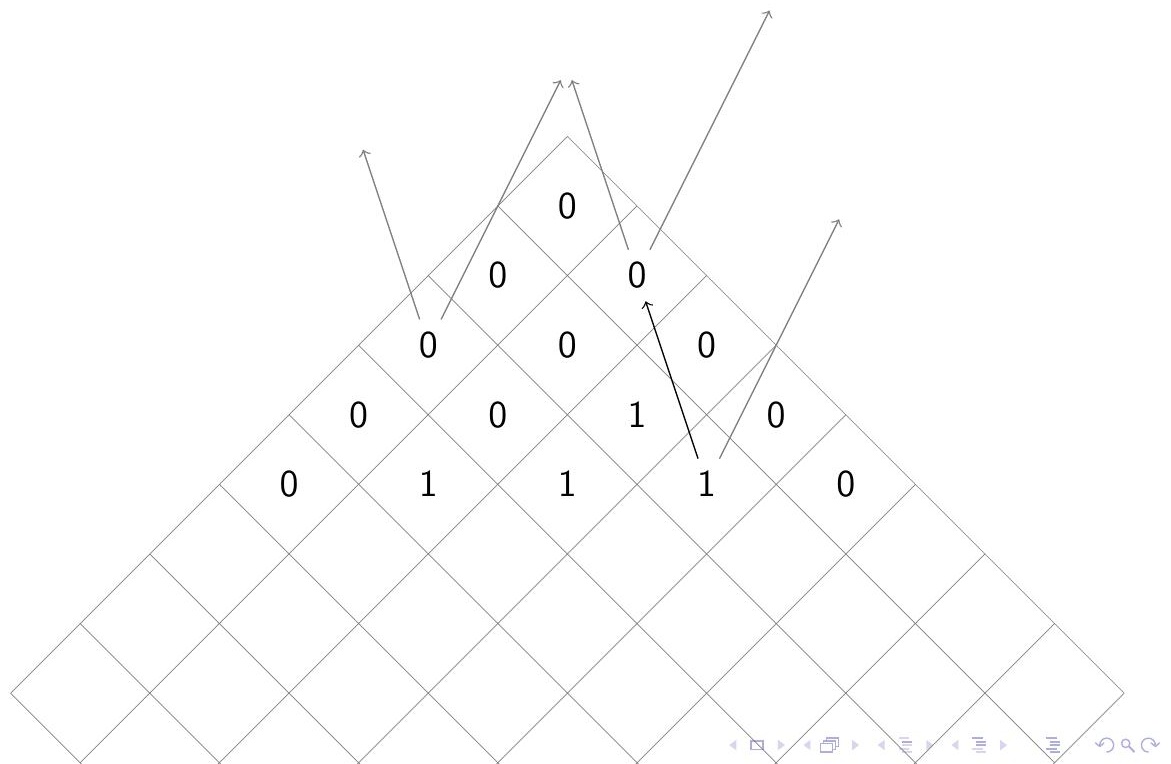


Cellular automata - kind of space-time diagram with bounded memory, the rules are $(1,2)$ and $(3,1)$

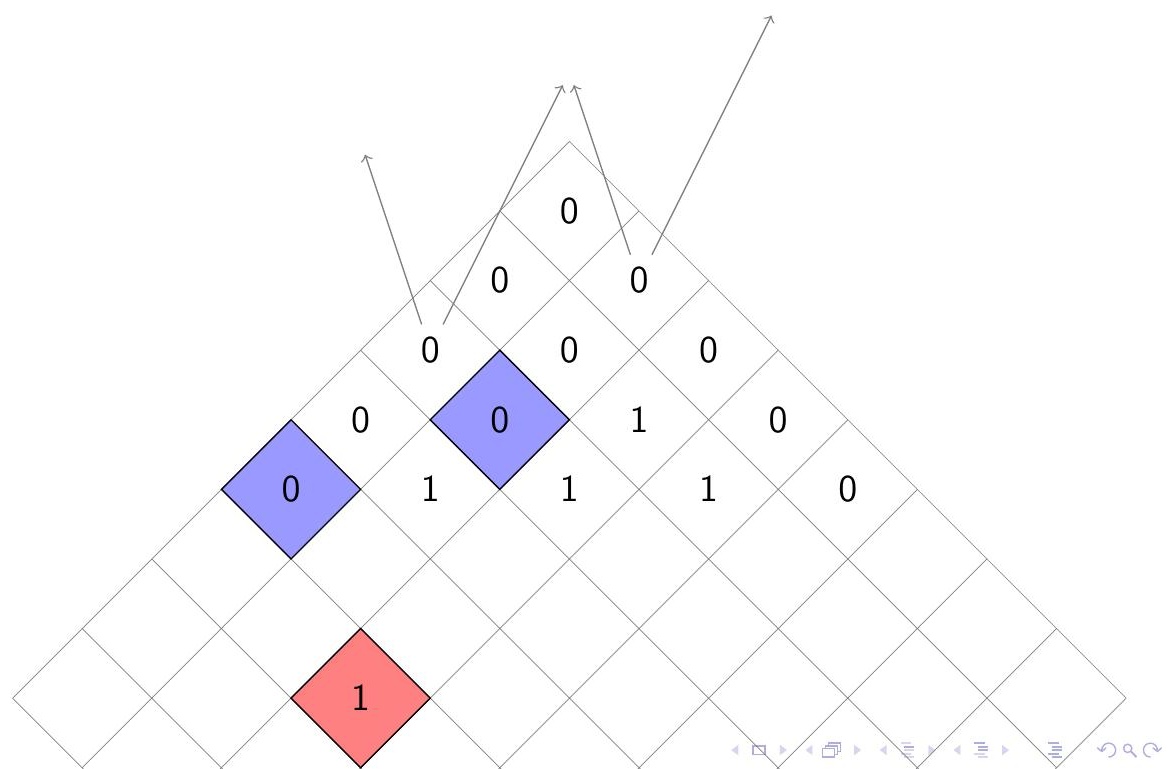


Cellular automata - kind of space-time diagram with bounded memory, the rules are $(1,2)$ and $(3,1)$

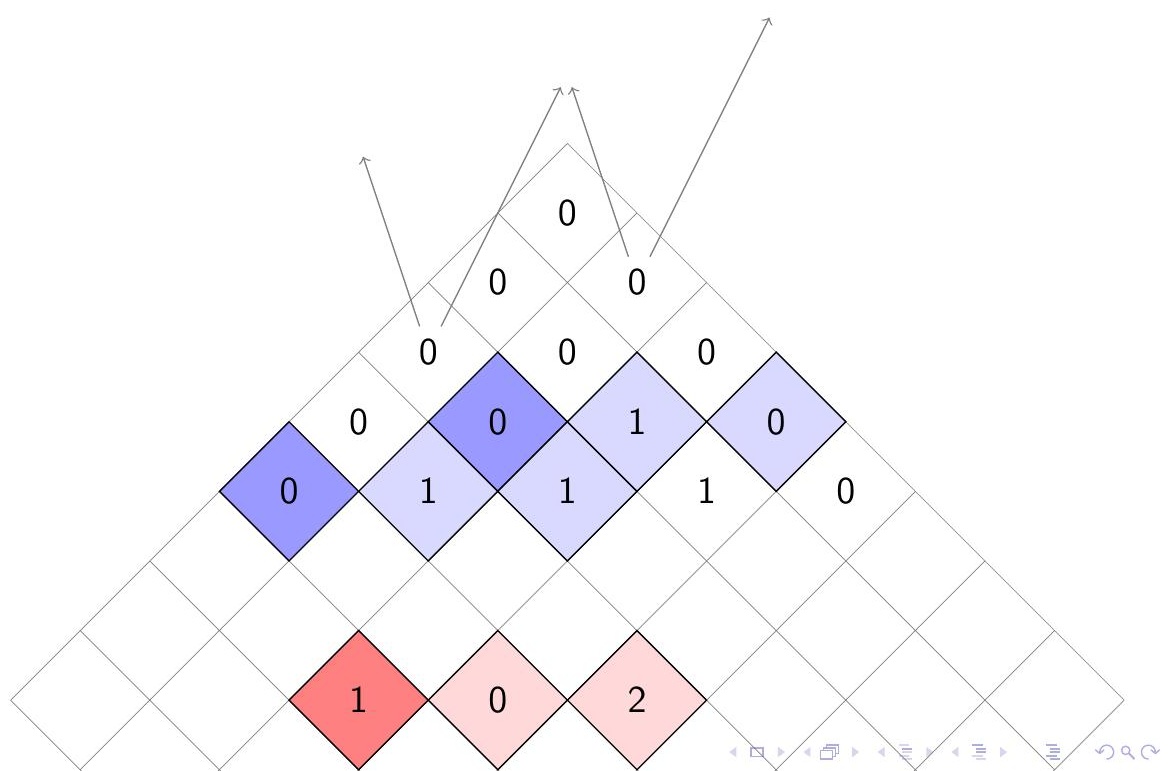


Bibliography (in chronological order of the slides)

A. Siegel, Combinatorial game theory, Graduate Studies in Mathematics, 146, AMS (2013).

E. R. Berlekamp, J. H. Conway, R. K. Guy, Winning ways for your mathematical plays, A K Peters, Ltd. (2001).

T. S. Ferguson, Game Theory, UCLA.

C. Berge, Graphes et hypergraphes, Monographies Universitaires de Mathématiques, No. 37. Dunod, Paris, (1970).

W. A. Wythoff, A modification of the game of Nim, Nieuw Arch. Wiskd. 7 (1907), 199-202.

圈 C. L. Bouton, Nim, a game with a complete mathematical theory, Ann. of Math. (2) 3 (1901/02), no. 1-4, 35-39.

J.-P. Allouche, J. Shallit, Automatic sequences. Theory, applications, generalizations. Cambridge Univ. Press, Cambridge, (2003). 
A. Cobham 1972, Uniform tag sequences, Math. Systems Theory 6 (1972), 164-192.

J.R. Büchi, Weak second-order arithmetic and finite automata, Z. Math. Logik Grundlag. Math. 6 (1960), 66-92.

V. Bruyère, G. Hansel, C. Michaux, R. Villemaire, Logic and p-recognizable sets of integers, Bull. Belg. Math. Soc. Simon Stevin 1 (1994), no. 2, 191-238.

M. Rigo, Formal Languages, Automata and Numeration Systems, vol. 2, Applications to recognizability and decidability. Networks and Telecommunications Series. ISTE, London; John Wiley \& Sons, Inc., Hoboken, NJ, (2014).

圆 G. Christol, Ensembles presque périodiques k-reconnaissables, Theoret. Comput. Sci. 9 (1979), 141-145.

G. Christol, T. Kamae, M. Mendès France, G. Rauzy, Suites algébriques, automates et substitutions, Bull. Soc. Math. France 108 (1980), 401-419. 
E. Rowland, R. Yassawi, A characterization of $p$-automatic sequences as columns of linear cellular automata, Adv. in Appl. Math. 63 (2015), 68-89.

S. Eilenberg, Automata, Languages and Machines, Vol. A, Academic Press, New-York (1974).

J.-P. Allouche, J. Shallit, The ring of $k$-regular sequences, Theoret. Comput. Sci. 98 (1991), 163-197.

A. Carpi, V. D'Alonzo, On factors of synchronized sequences, Theoret. Comput. Sci. 411 (2010), no. 44-46, 3932-3937.

嘈 J. Berstel, C. Reutenauer, Noncommutative rational series with applications, Encyclopedia of Mathematics and its Applications, vol. 137, Cambridge Univ. Press (2011).

A. Thue, Über unendliche Zeichenreihen, Norske vid. Selsk. Skr. Mat. Nat. KI. 7 (1906), 1-22. 
A. Thue, Über die gegenseitige Lage gleicher Teile gewisser Zeichenreihen, Norske vid. Selsk. Skr. Mat. Nat. KI. 1 (1912), $1-67$.

M. Lothaire, Combinatorics on Words, Encyclopedia of Mathematics and Its Applications, vol. 17, Addison-Wesley (1983).

圆 V. Keränen, Abelian squares are avoidable on 4 letters, ICALP, pp. 41-52 (1992).

(1) J. Cassaigne, J. D. Currie, L. Schaeffer, J. Shallit, Avoiding Three Consecutive Blocks of the Same Size and Same Sum, J. ACM 61 (2014), no. 2, Art. 10, 17 pp.

E. Prouhet, Mémoire sur quelques relations entre les puissances de nombres, C. R. Acad. Sci. Paris Sér. I 33 (1851), 225.

P. Borwein, C. Ingalls, The Prouhet-Tarry-Escott problem revisited, Enseign. Math. 40 (1994), 3-27. 
圆 J.-P. Allouche, J. Shallit, The ubiquitous Prouhet-Thue-Morse sequence, Sequences and their applications (Singapore, 1998), 1-16, Springer Ser. Dis- crete Math. Theor. Comput. Sci., Springer, London, (1999).

U. Blass, A.S. Fraenkel, The Sprague-Grundy function for Wythoff's game, Theoret. Comput. Sci. 75 (1990), no. 3, 311-333.

(-) Jiao, On the Sprague-Grundy values of the $\mathcal{F}$-Wythoff game. Electron, J. Combin. 20 (2013).

A. Gu, Sprague-Grundy values of the $\mathcal{R}$-Wythoff game, Electron. J. Combin. 22 (2015).

M. Weinstein, Invariance of the Sprague-Grundy function for variants of Wythoff's game, Integers 16 (2016).

A. Dress, A. Flammenkamp and N. Pink, Additive periodicity of the Sprague-Grundy function of certain Nim games, Adv. in Appl. Math. 22, 249-270 (1999). 
H. A. Landman, A simple FSM ${ }^{5}$-based proof of the additive periodicity of the Sprague-Grundy function of Wythoff's game, More Games of No Chance, 2002.

S. Beatty, Problem 3173, American Mathematical Monthly 33, 159.

M. Lothaire, Algebraic Combinatorics on Words, Encyclopedia of Mathematics and its Applications, vol. 90, Cambridge Univ. Press (2002).

圆 É. Zeckendorf, Représentation des nombres naturels par une somme des nombres de Fibonacci ou de nombres de Lucas, Bull. Soc. Roy. Sci. Liège 41 (1972) 179-182.

圆 J. Shallit, A generalization of automatic sequences, Theoret. Comput. Sci. 61 (1988), no. 1, 1-16.

國 J.-P. Allouche, E. Cateland, W. J. Gilbert, H.-O. Peitgen, J. Shallit, G. Skordev, Automatic maps in exotic numeration systems, Theory Comput. Syst. 30 (1997), no. 3, 285-331. 
J.-P. Allouche, K. Scheicher, R. Tichy, Regular maps in generalized number systems, Math. Slovaca 50 (2000), no. 1, 41-58.

M. Rigo, Generalization of automatic sequences for numeration systems on a regular language, Theoret. Comput. Sci. 244 (2000), no. 1-2, 271-281.

A. Maes, M. Rigo, More on generalized automatic sequences, J. Autom. Lang. Comb. 7 (2002), no. 3, 351-376.

Ch. Frougny, On the sequentiality of the successor function, Inform. and Comput. 139 (1997) 17-28.

A.S. Fraenkel, How to beat your Wythoff games' opponent on three fronts, Amer. Math. Monthly 89 (1982), no. 6, 353-361.

E. Duchêne, A.S. Fraenkel, R. Nowakowski, M. Rigo, Extensions and restrictions of Wythoff's game preserving its $\mathcal{P}$-positions, J. Combin. Theory Ser. A 117 (2010), no. 5, 545-567. 
O. Salon, Suites automatiques à multi-indices, Séminaire de théorie des nombres, Bordeaux, 1986-1987, exposé 4.

A. Maes, Morphisms and almost-periodicity, Discrete Appl. Math. 86 (1998), no. 2-3, 233-248.

A. Maes, An automata-theoretic decidability proof for first-order theory of $\langle N,\langle, P\rangle$ with morphic predicate $P, J$. Autom. Lang. Comb. 4 (1999), no. 3, 229-245.

A. Maes, Morphic predicates and applications to the decidability of arithmetic theories, UMH Univ. Mons-Hainaut, Ph.D. thesis, January 1999.

E. Duchêne, M. Rigo, Invariant games, Theoret. Comput. Sci 411 (2010), 3169-3180.

E. Duchêne, A. Parreau, M. Rigo, Deciding game invariance, Inform. and Comput. 253 (2017), 127-142.

涯 P. Lecomte, M. Rigo, Numeration systems on a regular language, Theory Comput. Syst. 34 (2001), 27--44. 
圆 G. Katona, A theorem on finite sets, Theory of Graphs, Proc. Colloquium, Tihany, Hungary (1966), 187-207.

D. H. Lehmer, The machine tools of combinatorics, in Applied Combinatorial Mathematics (E. F. Beckenbach Ed.), Wiley, New York, (1964), 5-31.

J. S. Lew, L. B. Morales, A. Sánchez-Flores, Diagonal polynomials for small dimensions, Math. Systems Theory 29 (1996), 305-310.

圆 É. Charlier, T. Kärki, M. Rigo, Multidimensional generalized automatic sequences and shape-symmetric morphic words, Discrete Math. 310 (2010), no. 6-7, 1238-1252.

R. K. Guy, C. A. B. Smith, The $G$-values of various games, Proc. Cambridge Philos. Soc. 52 (1956), 514-526.

R. B. Austin, Impartial and partisan games, Master thesis, Univ. of Calgary (1976). 